\title{
Numerical and experimental investigation of oblique shock wave reflection off a water wedge
}

\author{
Q. Wan ${ }^{1}$, H. Jeon ${ }^{1}$, R. Deiterding ${ }^{2}$, and V. Eliasson ${ }^{1,3} \dagger$ \\ ${ }^{1}$ Aerospace and Mechanical Engineering, University of Southern California, Los Angeles, CA \\ 90089-1189, USA \\ ${ }^{2}$ Aerodynamics and Flight Mechanics Research Group, University of Southampton, \\ Southampton SO17 1BJ, UK \\ ${ }^{3}$ Department of Structural Engineering, University of California, San Diego, La Jolla, CA \\ 92093-0085, USA
}

(Received $\mathrm{xx}$; revised $\mathrm{xx}$; accepted $\mathrm{xx}$ )

Shock wave interaction with solid wedges has been an area of much research in past decades, but so far, very few results have been obtained for shock wave reflection off liquid wedges. In this study, numerical simulations are performed using the inviscid Euler equations and the stiffened gas equation of state to study the transition angles, reflection patterns, and triple point trajectory angles of shock reflection off solid and water wedges. Experiments using an inclined shock tube are also performed and schlieren photography results are compared to simulations. Results show that the transition angles for the water wedge cases are within $5.3 \%$ and $9.2 \%$, for simulations and experiments respectively, compared to results obtained with the theoretical detachment criterion for solid surfaces. Triple point trajectory angles are measured and compared with analytic solutions, agreement within $1.3^{\circ}$ is shown for the water wedge cases. The transmitted wave in the water observed in the simulation is quantitatively studied, and two different scenarios are found. For low incident shock Mach numbers, $M_{s}=1.2$ and 2 , no shock wave is formed in the water but a precursor wave is induced ahead of the incident shock wave and passes the information from the water back into the air. For high incident shock Mach numbers, $M_{s}=3$ and 4 , precursor waves no longer appear but instead a shock wave is formed in the water and attached to the Mach stem at every instant. The temperature field in the water is measured in the simulation. For strong incident shock waves, e.g., $M_{s}=4$, the temperature increment in the water is up to $7.3 \mathrm{~K}$.

\section{Introduction}

Although the shock wave reflection phenomenon was discovered over a century ago (Mach 1878), this is still an active research area within the shock wave community. In general, when a planar shock wave meets a sharp, compressive, and straight solid corner, the resulting shock reflection configurations can be divided into two categories, regular reflection and irregular reflection, depending on the incident shock wave Mach number, $M_{s}$, reflection wedge angle, $\theta_{w}$, and gas specific heat ratio, $\gamma$. Figure 1 shows two types of reflection patterns, regular and irregular reflections, where two kinds of irregular reflections, single-Mach reflection and double-Mach reflection, are presented (Ben-Dor \& Takayama 1992; Ben-Dor 2007). In figure 1, $w$ and $s$ denote the horizontal and vertical

$\dagger$ Email address for correspondence: eliasson@ucsd.edu 


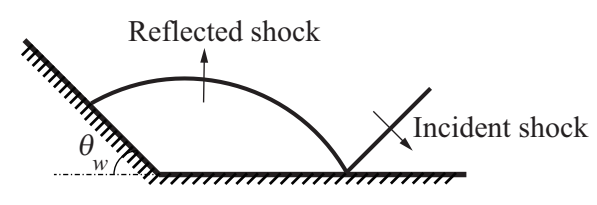

(a) Regular reflection

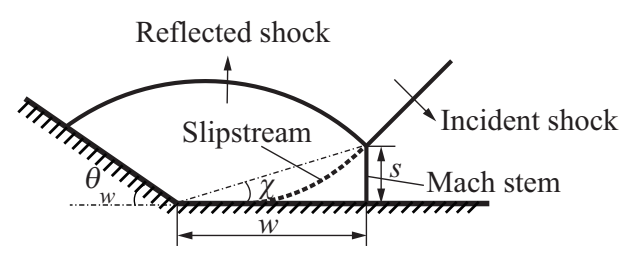

(b) Irregular reflection: single-Mach reflection

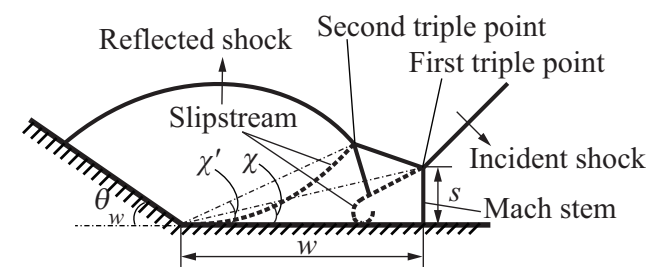

(c) Irregular reflection: double-Mach reflection

FiguRE 1. Illustration of two types of shock wave reflection off solid wedges, regular and irregular reflections, where two kinds of irregular reflections are shown. (a) Regular reflection; (b) Irregular reflection: single-Mach reflection; (c) Irregular reflection: double-Mach reflection.

displacements of the triple points, $\chi$ denotes the first triple point trajectory angle, and $\chi^{\prime}$ denotes the second triple point trajectory angle.

Shock reflection patterns can be analyzed in terms of the transition status and the reflection configuration. The transition status can be quantified using the transition angle, which determines when the reflection configuration transforms from irregular to regular. The reflection configuration can be described using the triple point trajectories. Using numerical methods, a high level of resolution can nowadays be achieved and can facilitate analysis of transition status and reflection configuration. Further, in shock wave experiments, the maximally achievable Mach number regimes can be limited due to the experimental setup, while in simulations, such limitation does not exist. Thus, numerical simulations provide a practical approach to investigate shock wave reflection phenomena.

Various experimental visualization techniques have been performed in shock tube setups to determine the transition angle using either high temporal imaging or spatial imaging (Geva et al. 1990; Kleine et al. 2014; Mouton 2006; Naidoo \& Skews 2011; Ram et al. 2015; Skews \& Kleine 2010; Skews \& Blitterswijk 2011). Technological advances of high temporal imaging equipment have made these systems readily available to many researchers. However, photographs from high-speed cameras often result in relatively low resolution due to high frame rates. Therefore, high temporal imaging can be used to understand succinct details of the entire process, but high spatial imaging will ultimately 
be required to provide accurate information (Versluis 2013). Nevertheless, studies to determine the transition angle using a combination of both high temporal and high spatial imaging are still lacking. Accordingly, in the current study, schlieren visualization with both high spatial and high temporal resolution are used to find the transition angles.

\subsection{Shock reflection off solid wedge}

Though much attention has been given to establishing a transition criterion from regular to irregular reflections, work is still ongoing because of unsatisfactory comparison between numerical and experimental findings with theoretical solutions. Theoretical solutions are based on inviscid Euler analysis, while in experiments researchers must deal with viscosity and surface roughness of the reflecting surface. One widely used transition criterion for regular to irregular reflection is the detachment criterion, originally proposed by von Neumann $(1943 a, b)$. The detachment criterion (von Neumann 1943a) is an analytic solution of the transition angle for shock reflection off solid wedges in inviscid flow. The detachment criterion predicts that transition from regular to irregular reflection occurs when the maximum flow deflection angle across the reflected shock is achieved and the regular reflection is no longer possible. The detachment criterion states that in steady flow, the flow velocities ahead of the incident shock and behind the reflected shock remain parallel while the flow deflection by the reflected shock wave is maximal.

In shock wave reflection configurations of pseudo-steady cases, the appearance of Mach stems and slipstreams can be an important feature to distinguish between regular and irregular reflection. Usually, at small wedge angles, the reflection configurations remain irregular, where Mach stems and slipstreams are clearly seen. While approaching the transition condition by, for example, increasing the inclination angle of the wedge, the length of the Mach stem decreases as the triple point moves closer to the reflecting surface. As the inclination angle is increased to a certain angle, the reflection configuration transitions from irregular to regular reflection. The critical $\theta_{w}$ at the transition status between irregular to regular reflection is referred to as the transition angle.

Shock wave reflection over solid wedges has been investigated previously by numerous research groups (Ben-Dor et al. 1987; Hornung et al. 1979; Hornung \& Taylor 1982; Hornung \& Robinson 1982; Mach 1878; von Neumann 1943a,b; Skews 2005; Onodera \& Takayama 1990; Sasoh et al. 1992). Hornung \& Taylor (1982) experimentally explored the influence of viscosity on the transition from regular to Mach reflection of strong shock waves $\left(M_{s}=5.5\right)$. Argon was used to avoid real-gas effects, and results showed that in pseudo-steady flows viscosity plays an important role such that the transition angle was affected by up to $7^{\circ}$ compared to the case with no viscosity.

One major difference between a solid and a water wedge is that, compared to solid wedges, the surface of a water wedge can be treated as perfectly smooth. However, the surface roughness for a solid wedge may be hard to quantify due to uncertainties in measurements. To quantify the effect of surface roughness, Ben-Dor et al. (1987) proposed a boundary-layer displacement-thickness model for predicting regular to Mach reflection transition. The model is specified for incident shock Mach numbers in the range of $1<M_{s}<2$, and surface roughness heights up to $2 \mathrm{~mm}$. Results showed that the flow behind the reflection point must be parallel to the average slope of the displaced surface. Onodera \& Takayama (1990) used both numerical and experimental methods to study the effect of slitted wedges on shock wave transition. Incident Mach numbers ranged from 1.07 to 3.03 , and slitted wedges with perforation ratios 0.34 and 0.4 were used. Perforation ratio is defined as the ratio of the perforated and overall surface area. Results showed that for stronger shock waves and a perforation ratio of 0.4 , the transition angle was decreased by about $10^{\circ}$ as compared with the detachment criterion. The numerical results agreed 
well with experimental results. More recently, Skews (2005) experimentally extended this study to a wider range of plate geometries. It was found that the plate thickness had a negligible influence on the results for the tested parameter range.

Shock wave reflection off curved surfaces have also been an area of interest. Kleine et al. (2014) experimentally and numerically investigated the influence of the Reynolds number on the triple point trajectories where a planar shock wave is reflected by cylindrical surfaces. Results showed that the delay of RR-MR transition due to viscous effects is low under high Reynolds numbers. More recently, Soni et al. (2017) performed numerical simulations to study the wave configurations related to the shock reflection over doubleconcave cylindrical surfaces. The study covered different incident shock Mach numbers as well as geometrical parameters of the reflectors, such as the radii of curvature and the initial wedge angles. Noticeable differences can be found between the shock patterns over the two adjacent reflectors of the same shape, where the shock reflection off the second reflector is strongly dependent on how fast the diffracted shock reaches the incident shock wave.

\subsection{Triple point trajectories}

The reflection configurations of single-Mach reflection and double-Mach reflection can be quantified by tracking the triple point trajectories. For single-Mach reflection, based on the assumptions that the shock wave configuration is self-similar and the triple point trajectories are straight lines emanating from the compressive corner, an analytic formulation (Ben-Dor \& Glass 1979, 1980; Ben-Dor 1978; Law 1970) was developed to describe the motion of the triple point trajectory angle, which is illustrated by $\chi$ in figure 1(b). Assumptions include a straight Mach stem normal to the wedge surface and uniform inviscid flow behind incident shock wave, reflected shock wave and Mach stem. The method to solve for the triple point trajectory angle in single-Mach reflection also applies for the first triple point in the case of a double-Mach reflection, $\chi$ in figure 1(c).

For the second triple point trajectory angle, denoted $\chi^{\prime}$ in figure 1(c), Ben-Dor (1980, 1981) formulated an analytic solution to describe the relative motion of the second triple point in terms of the first triple point. They also concluded that the triple point trajectory angle of the second triple point should be always greater than the first one. Later, Li \& Ben-Dor (1995) proposed a new analytic model to predict the second triple point trajectory of a double-Mach reflection. The new model improved the prediction tremendously by replacing the Law-Glass assumption by models accounting for the interaction of the shock wave reflection and the shock induced flow deflection processes.

\subsection{Shock interaction with liquid}

Both experiments and numerical simulations of a planar shock wave interaction with a liquid droplet have been performed by Igra \& Takayama (2001a,b). They proposed a numerical scheme to achieve a sharp interface between the gas and liquid phase. The Tait's equation of state was employed for water with $n=7.415$ and $B=296.3 \mathrm{MPa}$. With that scheme, shock wave interaction with a cylindrical water column was modeled and good agreement was shown compared with experimental interferogram results. More recently, Meng \& Colonius (2015) have numerically modeled the early stage of the breakup of water cylinders in the flow behind normal shock waves. The simulations were performed in a two-dimensional domain with symmetric boundary condition, where the stiffened gas equation of state was used to model both gases and liquids. Numerical schlieren images were compared with previous experimental findings (Igra \& Takayama 2001a), and agreement was shown regarding the breakup process of the cylinders. In 
particular, the water cylinder was first compressed in the streamwise direction and then extended in its spanwise dimension.

Planar shock wave reflection over water wedges has been experimentally studied by Takayama \& Ben-Dor (1989). In their study, experiments were conducted using an inclined shock tube that could be tilted from a horizontal to a vertical direction with an accuracy of $0.1^{\circ}$. Four different types of reflection configurations were observed: regular reflection, single-Mach reflection, transitional-Mach reflection, and double-Mach reflection. Measurements of the transition angles showed good agreement with the detachment criterion for the incident shock wave Mach number of the range $M_{s}>1.47$, and poor agreement for $M_{s}<1.47$. The theory of irregular reflection from von Neumann (1943a), also called three-shock theory, showed good agreement for strong shocks, i.e., $M_{s}>1.47$ (Colella \& Henderson 1990), but for weak shocks, the three-shock theory showed that the Mach reflection is physically impossible for $M_{s}<1.035$. The discrepancy between the three-shock theory and experimental results was referred to as the von Neumann paradox (Birkhoff 1950). Colella \& Henderson (1990) experimentally and numerically showed that Mach reflection was still possible for weak shocks. It was later confirmed later by Marchiano et al. (2007), Baskar et al. (2007), Karzova et al. (2015) and Desjouy et al. (2016) that irregular reflection and establishment of Mach stem could occur even for very weak shocks, i.e., $1.0001<M_{s}<1.01$. Additionally, one likely reason for the poor agreement was that the detachment criterion assumed uniform flow states behind reflected shock wave and Mach stem, while this assumption was incorrect when subsonic flow regions were involved. However, apart from the study of Takayama \& Ben-Dor (1989), shock reflections over liquid surfaces remain largely unexplored. This may be the result of challenges associated with performing experiments on liquid boundaries, and performing numerical simulations featuring multiphase phenomena with a deforming free surface. To investigate shock wave reflections at an air-water interface, previous research analytically calculated the energy exchange during shock reflection off an airwater interface, and results showed that nearly all of the incident energy is reflected back due to the impedance mismatch between the air and water (Henderson et al. 1990; Sakurai 1974). Borisov et al. (1965) first proposed the existence of shock wave refraction when a shock wave is sliding over a water layer, and meanwhile a precursor shock is formed in the air. Later, the refraction phenomenon was confirmed experimentally by Teodorczyk \& Shepherd (2012). More recently, Rodriguez et al. (2016) experimentally studied shock waves sliding over an horizontal free water layer. Two incident shock Mach numbers, $M_{s}=1.11$ and 1.43 , were examined, and the incident shock wave was vertical compared to the water surface. Results show that the transmitted shock wave in the water is ahead of the shock wave in the air. Further, the observed compression wave in the water travels at the same speed as the shock wave in the air, which is slower than the speed of sound in water. Thus, the propagation of the compression wave is driven by the shock wave in the air.

\subsection{Equation of state}

To numerically model water, two kinds of equations of state are typically used; (1) Tait's equation of state, and (2) stiffened gas equation of state. Tait's equation of state, which is designed to describe the compressibility of water, has the form

$$
\frac{p+B(s)}{p_{0}+B(s)}=\left(\frac{\rho}{\rho_{0}}\right)^{n},
$$

where subscript 0 refers to ambient conditions, $s$ denotes entropy, $p$ pressure, and $\rho$ density. Some researchers state that $B(s)$ and $n$ can be treated as constants for pressures 
up to $10^{4} \mathrm{MPa}$ (Kedrinskii 2005). Therefore, $B$ and $n$ are chosen such that density, $\rho_{0}$, and speed of sound, $a_{0}$, are properly estimated through

$$
B=\rho_{0} a_{0}{ }^{2} / n, a_{0}=\sqrt{\frac{\gamma\left(p_{\infty}+p\right)}{\rho}} .
$$

The following numerical values are most commonly used in the Tait's equation of state, $B=296.3$, 304.7 and 321.4 MPa, and $n=7.415,7.15$ and 7 (Igra \& Takayama 2001a,b; Cole 1948; Ridah 1988). A detailed examination of equations and charts for shock waves in water has been produced by Ridah (1988), who used $n=7$ and $B=321.4 \mathrm{MPa}$ resulting in $a_{0}=1500 \mathrm{~m} / \mathrm{s}$.

The stiffened gas equation of state takes the form

$$
p=(\gamma-1) \rho e-\gamma p_{\infty},
$$

where $e$ is the specific total energy, and $\gamma$ and $p_{\infty}$ are two empirically determined constants. When $p_{\infty}=0$, the stiffened gas equation of state reverts to the perfect gas equation of state. Assuming a constant specific heat at constant volume, $c_{v}$, the internal energy in the stiffened gas model reads (Flåtten et al. 2011)

$$
e=c_{v} T+\frac{p_{\infty}}{\rho}
$$

which allows expressing Equation (1.3) in terms of the temperature as

$$
p+p_{\infty}=(\gamma-1) \rho c_{v} T
$$

The two constants are most often set to $p_{\infty}=600$ and $300 \mathrm{MPa}, \gamma=4.4,5.5$ and 7.0 (Grove \& Menikoff 1990; Saurel \& Abqrall 1999; Shyue 1999). Jolgam et al. (2012) used a few test problems to verify the performance of a proposed Eulerian numerical method simulating multi-phase flows, air and water, while simultaneously comparing the computational cost of different equations of state. Both stiffened gas and van der Waals equation of state were used for air, while stiffened gas and Tait's equation of state were utilized for water. Three test problems consisting of a water-air shock tube, a gas-air shock tube, and a water faucet test, were considered. Results showed that the van der Waals equation of state needed more time steps and CPU time than the stiffened gas equation of state to obtain the solution, while stiffened gas (with $\gamma=4.4$ and $p_{\infty}=600 \mathrm{MPa}$ ) and Tait's equation of state (with $n=7.15$ and $B=331 \mathrm{MPa}$ ) for water needed roughly the same number of time steps and the same CPU time. Moreover, in their study, the stiffened gas equation of state for water was defined using constants $\gamma=4.4$ and $p_{\infty}=600 \mathrm{MPa}$, while the Tait's equation of state was defined with $n=7.15$ and $B=331 \mathrm{MPa}$.

In this study, an inviscid shock-capturing multi-phase solver is used to simulate shock wave behavior at air-water interfaces in a two-dimensional domain. Six different incident shock Mach numbers, $M_{s}=1.2,1.38,1.52,2,3$, and 4, are used in the simulations and three different shock Mach numbers, $M_{s}=1.20,1.38$, and 1.52, are investigated in the experiments. Shock reflection solutions from water wedges are compared with those from solid wedges. In particular, transition angles from regular to irregular reflection for both water and solid wedges were obtained for a range of incident shock Mach numbers. Further, in order to quantify the reflection configuration, the trajectories of triple points in both single-Mach reflection and double-Mach reflection were measured. Finally, the results from the numerical simulations were compared to experimental data using schlieren visualization. 


\section{Numerical approach}

A two-dimensional flow field was considered, where for both solid and water wedge cases the wedge surface was impacted by a planar shock wave.

\subsection{Governing equations}

The set of used governing equations is based on a multi-component model that employs the phase volume fractions $\alpha^{i}$ with $\sum_{i=1}^{m} \alpha^{i}=1$. Mixture quantities are given as

$$
\rho=\sum_{i=1}^{m} \alpha^{i} \rho^{i}, \quad \rho \mathbf{u}=\sum_{i=1}^{m} \alpha^{i} \rho^{i} \mathbf{u}^{i}, \quad \rho e=\sum_{i=1}^{m} \alpha^{i} \rho^{i} e^{i} .
$$

In this description, multiple fluids are represented as mostly separated phases that can be described by a single set of two-dimensional inviscid Euler equations

$$
\begin{gathered}
\partial_{t} \rho+\nabla \cdot(\rho \mathbf{u})=0, \\
\partial_{t}(\rho \mathbf{u})+\nabla \cdot(\rho \mathbf{u} \otimes \mathbf{u})+\nabla p=0, \\
\partial_{t}(\rho E)+\nabla \cdot((\rho E+p) \mathbf{u})=0,
\end{gathered}
$$

which represent the conservation of mixture mass, mixture moment and total energy without any body forces. Here, $\mathbf{u}$ is the velocity vector and $E=e+\frac{1}{2} \mathbf{u}^{T} \mathbf{u}$ the specific total energy. Applying a stiffened gas equation of state

$$
p^{i}=\left(\gamma^{i}-1\right) \rho^{i} e^{i}-\gamma^{i} p_{\infty}^{i}=\left(\gamma^{i}-1\right) \rho^{i} c_{v}^{i} T^{i}-p_{\infty}^{i},
$$

the total hydrodynamic pressure $p$ and $p_{\infty}$ of the mixture are given as

$$
\frac{p}{\gamma-1}=\sum_{i=1}^{m} \frac{\alpha^{i} p^{i}}{\gamma^{i}-1}, \quad \frac{\gamma p_{\infty}}{\gamma-1}=\sum_{i=1}^{m} \frac{\alpha^{i} \gamma^{i} p_{\infty}^{i}}{\gamma^{i}-1} .
$$

Instead of using propagation equations of $\alpha^{i}$ to distinguish the components, the approach of Shyue (1998) has been adopted to supplement Equations (2.2)-(2.4) with the two advection equations

$$
\begin{aligned}
& \frac{\partial}{\partial t}\left(\frac{1}{\gamma-1}\right)+\mathbf{u} \cdot \nabla\left(\frac{1}{\gamma-1}\right)=0, \\
& \frac{\partial}{\partial t}\left(\frac{\gamma p_{\infty}}{\gamma-1}\right)+\mathbf{u} \cdot \nabla\left(\frac{\gamma p_{\infty}}{\gamma-1}\right)=0 .
\end{aligned}
$$

Although this description is applicable only to two-component systems, i.e., $m=2$, the benefit is that the direct utilization of Equations (2.7), (2.8) in the governing equations and therefore direct discretization together with (2.2)-(2.4) is the simplest remedy (Shyue 2006) to the otherwise intrinsic problem of nonphysical numerical pressure oscillations at interfaces with vastly different parameters $\gamma^{i}$ and $p_{\infty}^{i}$ (Abgrall \& Karni 2001). Here, the parameters for water were defined as $\gamma^{W}=7.0$ and $p_{\infty}^{W}=300 \mathrm{MPa}$ (Grove \& Menikoff 1990; Shyue 1999), resulting in an ambient speed of sound of $a_{0}=1452 \mathrm{~m} / \mathrm{s}$ and $\gamma^{A}=1.4$ and $p_{\infty}^{A}=0$ for air.

\subsection{Numerical method}

A time-explicit finite volume approach is used to discretize the governing equations with the second-order accurate Wave Propagation Method (LeVeque 2002). This method is designed for schemes in flux-difference splitting form and can handle the conservative equations (2.2)-(2.4) with an approximate Riemann solver as well as the non-conservative 
advection equations (2.7) and (2.8). In order to cope with realistic density differences across the air-water interface, the HLLC scheme by Toro et al. (1994) is used as approximate Riemann solver. An extended version of the HLLC scheme, tailored for the above set of governing equations for two-phase flow and smoothly incorporated into the Wave Propagation approach had been specially derived (Deiterding et al. 2009). Thanks to the robustness of the HLLC approach, the subsequent computations could use the accurate densities $\rho^{W}=997 \mathrm{~kg} / \mathrm{m}^{3}$ and $\rho^{A}=1.18 \mathrm{~kg} / \mathrm{m}^{3}$.

In order to enable technically relevant computations, the sketched finite volume method has been incorporated into the Cartesian adaptive mesh refinement solver system AMROC V2.0 (Deiterding 2011) within the freely available fluid-structure coupling software Virtual Test Facility (Deiterding et al. 2006). The consideration of geometrically complex boundaries in AMROC is achieved by a generic and discretization-independent level set technique. Based on signed distance information stored in the level set function, reflective wall boundary conditions are constructed by inter- and extrapolation operations in cells adjacent to the boundary, but deemed outside of the fluid domain, before the Cartesian discretization is employed to compute the next time step (Deiterding 2009). Block-based mesh refinement is applied and regenerated consecutively at runtime to mitigate inaccuracies from the boundary approximation as well as to capture essential flow features, like shock waves and material interfaces with increased resolution (Deiterding 2011). Time step refinement by the same factor as the spatial refinement ensures that the stability condition of the explicit finite volume scheme is in principle satisfied on all refinement levels.

Validation simulations for the sketched dynamically adaptive two-phase HLLC method are provided by Perotti et al. (2013), where elastic deformations of tubes from shock waves in water have been analyzed meticulously. Further verification and validation simulations for water-hammer and underwater explosion driven plastic deformation and rupture of metallic plates with two-phase flow and empirical cavitation modeling are given by Cirak et al. (2007) and Deiterding et al. (2009).

\subsection{Simulation setup}

The simulation time for each case is chosen such that the incident shock wave has enough time to impact the air-water interface and reach the corner formed by the wedge and the outer solid boundary and reflect back. During this time period, the displacement of fluid particles due to gravity is negligible. The geometrical setup for the water and solid wedge cases is shown in figures $2(\mathrm{a})$ and (b), respectively. The rectangular outer frames represent the boundaries of the computational domains, while the oblique thick lines denote the boundary of the test section. In figure $2(\mathrm{a})$, the shape of the geometrical setup is chosen to match an experimental setup to enable comparison of experimental and numerical results. Results from shock reflection off a liquid wedge were compared to those off a solid wedge. The inclination angle of the wedge is denoted $\theta_{w}$, see figures 2 (a) and (b). The boundary condition of the upper left corner of the computational domain was setup as constant inlet flow, while the rest of the boundaries were modeled as rigid walls.

Cartesian grids were applied to the whole computational domain. For all the simulations, adaptive mesh refinement (AMR) was applied during the simulation. The dynamic mesh refinement was based on the scaled gradients of pressure, density and volume fraction $\alpha_{i}$ as well as of the level set representing the rigid boundaries. Three AMR

levels were chosen and a refinement factor of 2 was used. A coarse background grid of cell size $100 \times 100 \mu^{2}$ was created so after mesh refinement, the smallest effective cell size was $25 \times 25 \mu^{2}$. 


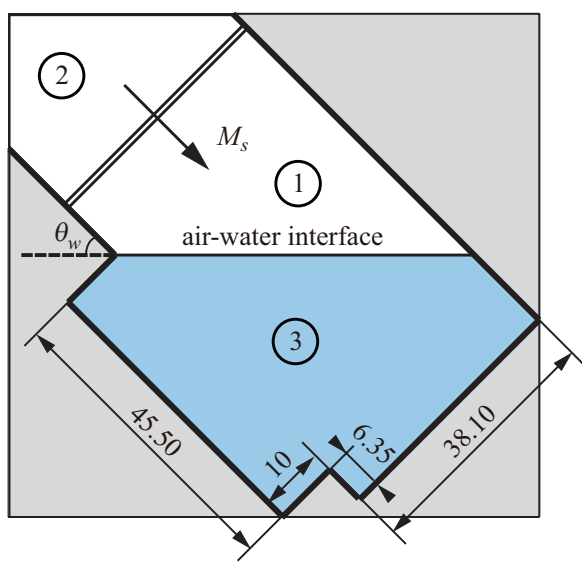

(a) Water wedge case

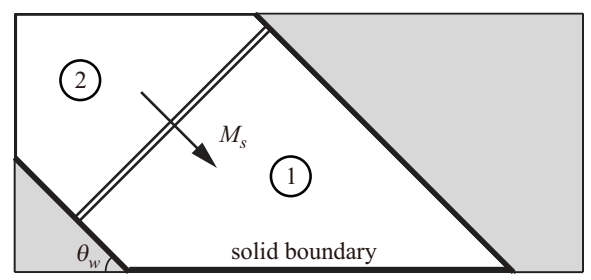

(b) Solid wedge case

FIGURE 2. Setup for numerical simulations of shock wave reflection off a (a) water wedge, and (b) solid wedge. Region 1 and 2 and are filled with air while region 3 is filled with water. Dimensions in $\mathrm{mm}$, not to scale.

Initial conditions ahead of the incident shock wave, denoted region 1 in both figures 2 (a) and (b), were set up with standard atmospheric conditions: $p_{1}=1.013 \times 10^{5} \mathrm{~Pa}, \rho_{1}=$ $1.18 \mathrm{~kg} / \mathrm{m}^{3}$ and $T_{1}=25^{\circ} \mathrm{C}$. The conditions behind the incident shock wave, region 2 in figure 2, were determined using normal shock relations. Region 3 shown in figure 2(a) was filled with water at atmospheric pressure and room temperature, same as region 1. The resulting density of water is $\rho_{3}=997 \mathrm{~kg} / \mathrm{m}^{3}$. All the simulations assumed inviscid flow and the solid wedge surface to be rigid and smooth.

Each simulation of a shock reflecting off water wedge took approximately $25 \mathrm{CPU}$ hours using a single core of $3.20 \mathrm{GHz}$ Intel(R) Core(TM) i7-3930K CPU, but only 11 CPU hours for shock reflection off solid wedge.

The transition angle measured through the simulations fell into a range with uncertainty of $1^{\circ}$ because the inclination wedge angle was increased by increments of $1^{\circ}$ after each run.

\subsection{Constraints of the numerical simulation}

Without the presence of viscosity, the shock reflection patterns are affected in two ways: (1) For irregular shock reflection, viscosity delays the establishment of the Mach stem. Kleine et al. (2014) confirmed that the Reynolds number mainly affects the initial establishment of the Mach stem and only to a lesser degree its subsequent growth. Thus, in terms of shock patterns, the Mach stem is established earlier without viscosity, but the triple point trajectory angle is not much affected; (2) Viscosity decreases the transition angle, and therefore simulation results in this study are compared with experiments, shock reflection over the solid wedge cases and the analytical solution under the same inviscid assumption.

The entire computational domain was meshed using Cartesian grid. Because of the nature of the Cartesian grid, when a shock wave, air-water interface or physical boundary is not aligned with the grid, assignment of the initial conditions to the corresponding cells 

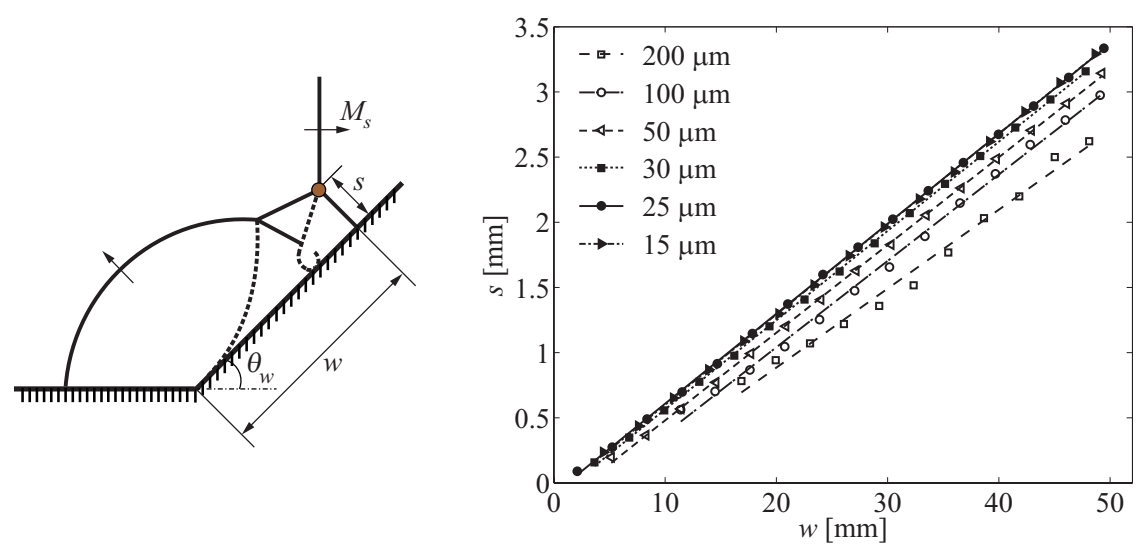

(a) First triple point trajectory
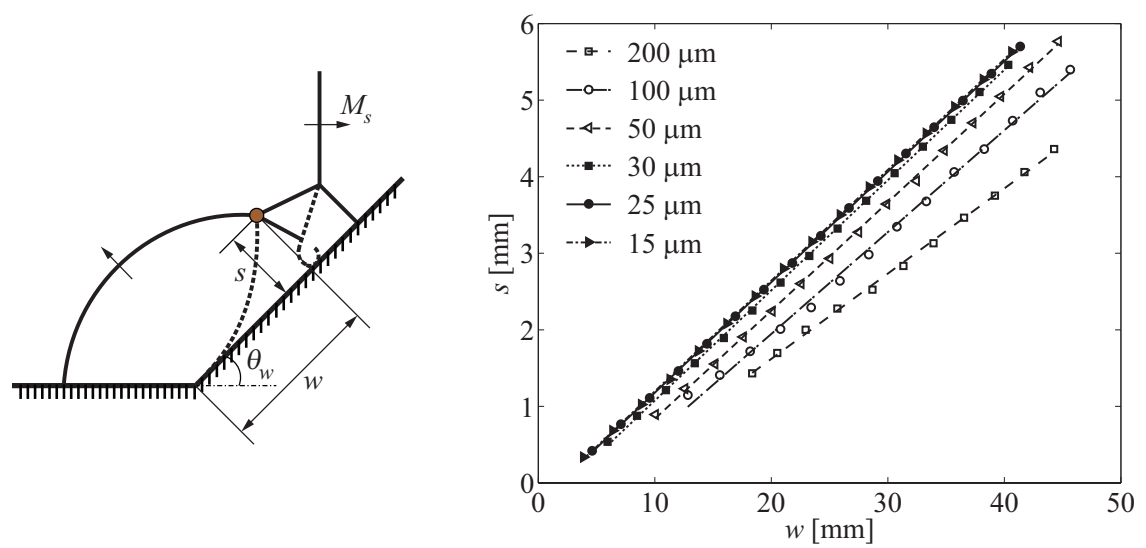

(b) Second triple point trajectory

Figure 3. Mach stem heights $s$ versus displacement in horizontal direction $w$ for the (a) first triple point and (b) second triple point at $M_{s}=3$ and $\theta_{w}=45^{\circ}$ solid wedge. Results obtained with different grid sizes.

results in a saw-tooth-shaped discontinuity or boundary, which leads to an addition of artificial roughness. Though the artificial roughness can be reduced by refining the grid size, it is never eliminated as long as the Cartesian mesh is used. The same grid effect can be found in the work of e.e. Soni et al. (2017). The artificial roughness may delay the establishment of Mach stem and therefore affect transition angles. Thus, a convergence test using six different mesh sizes, i.e., $200 \mu \mathrm{m}, 100 \mu \mathrm{m}, 50 \mu \mathrm{m}, 30 \mu \mathrm{m}, 25 \mu \mathrm{m}$, and $15 \mu \mathrm{m}$, with regard to the triple point trajectories is conducted for the solid wedge case with $M_{s}=3$ and $\theta_{w}=45^{\circ}$, as shown in figure 3 . The convergence study is used to prove that results of triple point trajectories are independent from grid size and the mesh is refined enough. In the convergence study, the angle formed between the wedge surface and the Cartesian grid is $45^{\circ}$. Results obtained from grid sizes $25 \mu \mathrm{m}$ and $15 \mu \mathrm{m}$ show negligible difference. Therefore, the grid size, $25 \mu \mathrm{m}$, is determined to be refined enough to capture the initiation of the Mach stem and this grid size is used in all the other shock wave reflection simulations.

The liquid surface tension is not considered in the simulations. However, the lack of 
surface tension does not affect the shock reflection patterns and corresponding results, since the deformation of the interface is negligible during the simulated time.

Due to the nature of the shock capturing scheme, shock waves and interfaces are diffusive and spread over several grid cells. Therefore, the mixture regions near the airwater interface do not indicate the molecular mixing of the two-phase flow, but are because of the non-physical numerical diffusion.

\section{Experimental approach}

Experiments are performed using an adjustable, inclined shock tube and schlieren visualization with either high spatial or high temporal resolution.

\subsection{Details of the shock tube}

The experimental setup uses an inclined shock tube of which the inclination angle can be adjusted, as shown in figure 4 . The shock tube was designed to study shock wave reflection off solid and liquid wedges, and as of such the inclination angle of the shock tube can be adjusted in the range $0^{\circ}<\theta_{w}<90^{\circ}$. The angle of the shock tube is controlled by moving a support arm along an Acme threaded rod, which travels $6.4 \mathrm{~mm}$ per rotation and the angle is measured by an inclinometer (Wixey WR365, $\pm 0.1^{\circ}$ accuracy). The shock tube consists of a driver section, a driven section with square interior sides, and a test section. The driver section of the inclined shock tube is $300 \mathrm{~mm}$ long and has a $73 \mathrm{~mm}$-diameter circular cross-section. The driven section and the test section both have square $38.1 \mathrm{~mm}$ inner sides. The length of the driven section is about 17 times longer than the side of the test section to ensure that the shock is planar upon arriving in the test section (Bleakney et al. 1949). A diaphragm is located between the driver and driven sections, and a shock wave can be generated after the diaphragm rupture by needle mechanism in the driver section (Jeon et al. 2015). Three different thicknesses of moisture-resistant Polyester film (12.7, 25.4, and $50.8 \mu \mathrm{m}$ thickness, $\pm 10 \%$ tolerance) were used as diaphragm materials to perform experiments featuring driver pressures of 138,310 , and $586 \mathrm{kPa}(20,45$, and $85 \mathrm{psi})$, respectively. The test section was designed as a "T" shape to minimize the meniscus between the water surface and the solid wall of the test section. The windows of the test section were made of $12.7 \mathrm{~mm}$ thick plexiglass. In the test section, two different types of reflecting surfaces are considered: water and solid wedges. Tap water is used at room temperature $(300 \mathrm{~K})$. The solid wedges are made of polycarbonate (Makrolon ${ }^{\circledR}$ GP sheet) with 10 different angles (from $40^{\circ}$ to $50^{\circ}$ ) and care is taken to ensure that the reflecting surface of the polycarbonate sheet has a surface roughness similar to glass.

All experiments were conducted with air as test gas at atmospheric pressure (101 $\mathrm{kPa})$ and room temperature $(300 \mathrm{~K})$. The shock Mach number was measured using three pressure transducers (S1 and S3: PCB 113B21, S2: PCB 113B31) placed in the driven section ahead of the test section, as shown in figure 4(b). The incident shock Mach number was determined using the elapsed time between the shock passing the three pressure transducers. The shock Mach numbers were measured with a standard deviation within $\pm 0.70 \%$ and a measurement uncertainty less than $\pm 0.80 \%$ in each experiment. After the pressure signals were recorded by the oscilloscope, a low pass Fourier filter was used to remove all frequencies above $100 \mathrm{kHz}$ for data recorded by $\mathrm{S} 1$ and $\mathrm{S} 3$, and $80 \mathrm{kHz}$ for data recorded by $\mathrm{S} 2$. The cutoff frequency was chosen such that it is below the natural frequency of the pressure transducer, which is $500 \mathrm{kHz}$ for S1 and S3, or $400 \mathrm{kHz}$ for S2. 


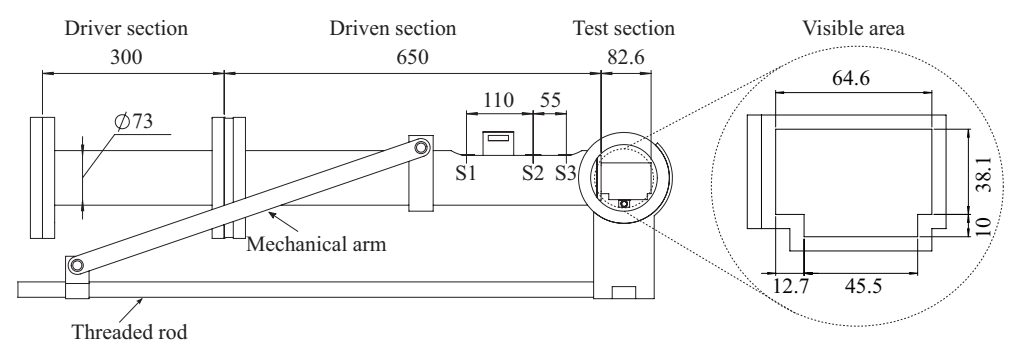

(a)

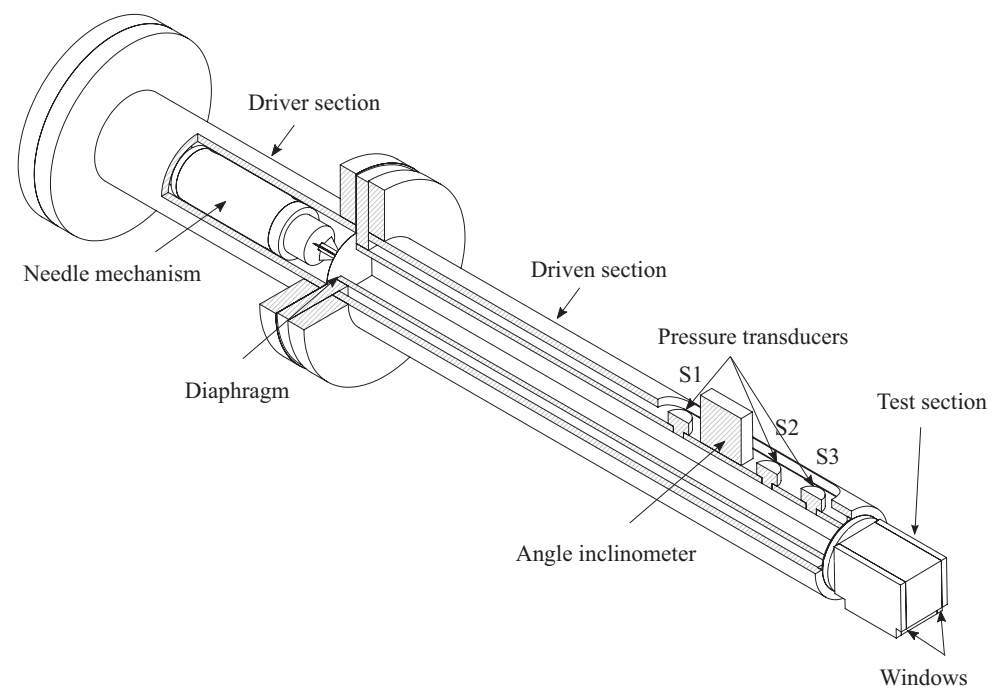

(b)

FiguRE 4. Schematic description of inclined shock tube: (a) side and cross view of the inclined shock tube. Dimensions in mm; (b) detailed cut-view of the inclined shock tube.

\subsection{Visualization setup}

For this study, schlieren visualization with both high spatial and high temporal resolution are used to compensate the respective drawbacks of the two techniques. Both methods are set up to avoid motion blur (Versluis 2013), which can be expressed as

$$
v \times \Delta t \leqslant \frac{P}{M_{\mathrm{im}}},
$$

when $v$ is an object's speed, $\Delta t$ is the exposure time of the camera or the light source, $P$ is the size of one pixel, and $M_{\mathrm{im}}$ is the image magnification factor. These two methods are summarized in Table 1 and explained in detail in the following two subsections. To obtain quantitative data from the images, optical distortions resulting from the visualization system are corrected using the control point tool box in MATLAB (Delpino Gonzales \& Eliasson 2015).

Typically, the thickness of the shock wave is on the order of the mean free path of the gas, which at standard atmospheric conditions results in $200 \mathrm{~nm}$ shock thickness (Fox et al. 1985). In practice, the thickness of the dark band representing the shock wave that is obtained by using schlieren photography can be affected by several factors such as the 
TABLE 1. Summary of camera settings.

\begin{tabular}{lcccccc}
\hline Camera & $\begin{array}{c}\text { Focal } \\
\text { length } \\
{[\mathrm{mm}]}\end{array}$ & Resolution & $\begin{array}{c}\text { Scale } \\
\text { factor } \\
{[\mu \mathrm{pix} / \mathrm{pix}]}\end{array}$ & $\begin{array}{c}\text { Exposure } \\
\text { time } \\
{[\mathrm{ns}]}\end{array}$ & $\begin{array}{c}\text { Frame } \\
\text { rate } \\
{[\mathrm{fps}]}\end{array}$ & $\begin{array}{c}\text { Interframe } \\
\text { time } \\
{[\mu \mathrm{s}]}\end{array}$ \\
\hline $\begin{array}{l}\text { Phantom V711 } \\
\text { Nikon D90 }\end{array}$ & 50 & $\begin{array}{c}176 \times 96 \\
4288 \times 2848\end{array}$ & $\begin{array}{c}450 \\
27\end{array}$ & $\begin{array}{c}294 \\
18\end{array}$ & $\begin{array}{c}200,000 \\
1\end{array}$ & $\begin{array}{c}5 \\
\mathrm{~N} / \mathrm{A}\end{array}$ \\
\hline
\end{tabular}

strength of the shock wave, a non-parallel incident light angle, and shadowgraph effects. In this experiment, when using the Phantom V711 camera, the thickness of the dark band was roughly equal to one pixel $(450 \mu \mathrm{m})$. However, when using the Nikon D90, it was about 10 pixels $(270 \mu \mathrm{m})$, similar to previous results (Ram et al. 2015).

\subsubsection{Schlieren visualization with high temporal resolution}

High temporal resolution was obtained using a z-folded schlieren setup (Wang \& Eliasson 2012) with a high-speed camera (Phantom V711) and a high-power lightemitting diode (Cree XLamp, XP-G2 LEDs, Cool white). Schlieren visualization was used instead of shadowgraph to avoid any inherent ambiguity from the shadow images. The knife-edge was placed vertically such that the schlieren images, which usually had a $50 \%$ cutoff, satisfied both resolution and sensitivity (Settles 2012). The high-speed camera was equipped with a $50 \mathrm{~mm}$ focal length lens (Nikkor $50 \mathrm{~mm} f / 1.4$ lens) to increase the frame rate since higher frame rates can be achieved by reducing the resolution. For this study, $176 \times 96$ pixels and 200,000 frames per second (fps) with a minimum exposure time of $294 \mathrm{~ns}$ were used. Precise triggering, with \pm 20 ns timing accuracy of the high-speed camera, was achieved by an output trigger signal sent from the pressure transducer, S1, to the high-speed camera. The signals from the pressure transducers were collected by a signal conditioner (PCB Piezotronics 482C), and recorded by a digital oscilloscope (LeCroy, Wave Surfer 24Xs-A). Figures 5 illustrates schlieren visualization with high temporal resolution, showing $\mathrm{RR}$ reflecting off the water with $M_{s}=1.52$ and $47^{\circ}$ deflection angle.

\subsubsection{Schlieren visualization with high spatial resolution}

Schlieren visualization with high spatial single-frame photographs were obtained using a Digital-Single Lens Reflex (DSLR) camera, a flash lamp (Nanolite KL-L Blitzlampe, High-speed Photo-system, \pm 10 ns timing accuracy) with an 18 ns duration, and a flash lamp driver (High-speed Photo-system). A Nikon D90 SLR camera was equipped with a $200 \mathrm{~mm}$ lens (AF Micro-Nikkor $200 \mathrm{~mm} f / 4$ D IF-ED) to produce high spatial resolution images of $4288 \times 2838$ pixels. The exposure time of the camera was set to 2.5 seconds, manually, in a dark-room setting. The timing of the flash lamp was controlled by a delay generator (BNC Model 575, \pm 0.8 ns trigger accuracy) operated in single pulse shot mode with different delay times. The trigger signal was sent from pressure transducer S1 and collected by the signal conditioner. Figure 6 represents schlieren visualization with high spatial resolution. Figure 6(a) and (b) show a regular reflection configuration over water. 


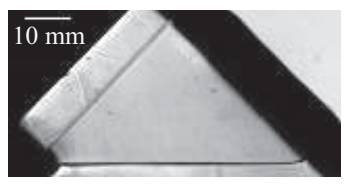

(a) $0 \mu \mathrm{s}$

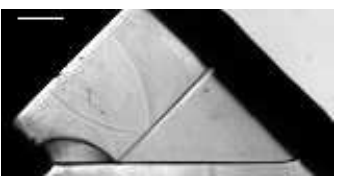

(d) $30 \mu \mathrm{s}$

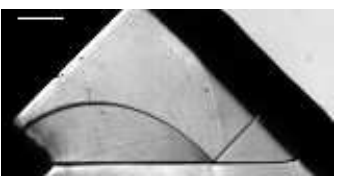

(g) $60 \mu \mathrm{s}$

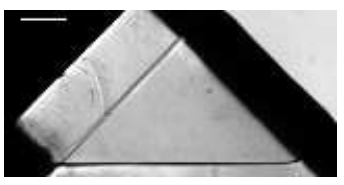

(b) $10 \mu \mathrm{s}$

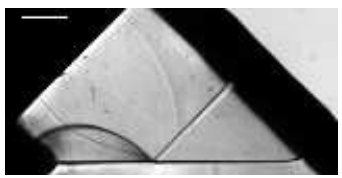

(e) $40 \mu \mathrm{s}$

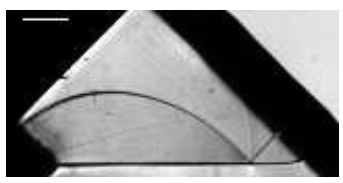

(h) $70 \mu \mathrm{s}$

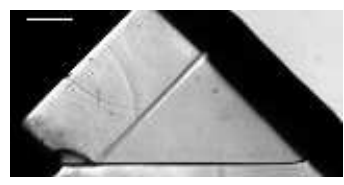

(c) $20 \mu \mathrm{s}$

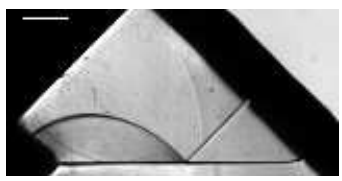

(f) $50 \mu \mathrm{s}$



(i) $80 \mu \mathrm{s}$

FiguRE 5. A series of schlieren photographs using a Phantom V711 camera showing shock reflection off a water surface at $M_{s}=1.52$ and $\theta_{w}=47^{\circ}$ resulting in regular reflection.

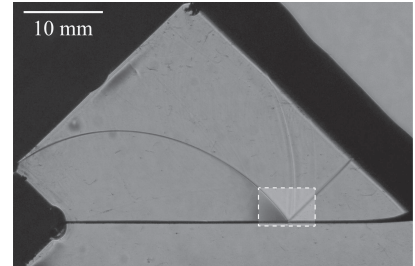

(a)

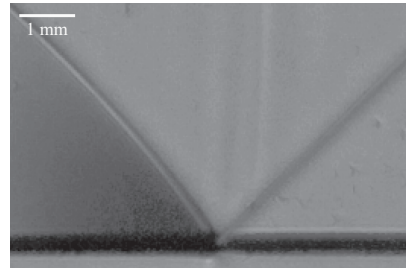

(b)

FiguRE 6. Schlieren photographs using a Nikon D90 camera showing (a) regular reflection off a water surface for $M_{s}=1.38$ and $\theta_{w}=45^{\circ}$ and (b) a magnified image of the reflecting surface highlighted by the dashed rectangle in (a).

\subsection{Experimental procedure}

The water in the test section was changed every time in between experiments. A hydrophobic coating (Rain-X, SOPUS Products) was used on the windows of the test section to minimize the wetting condition and to clean the windows. There exists a small angle between the solid and the tangent line to the liquid surface, a contact angle, in the test section due to the liquid surface tension, as shown in Fig. 7(a). Since the purpose of this study was to find transition angles, it was necessary to observe whether a Mach stem is present above the liquid surface. If a concave meniscus occurs, the free surface cannot be visualized. As such, the curvature of the liquid was maintained to be slightly convex by filling the test section with liquid from the bottom (Takayama \& Ben-Dor 1989). Figure 7(b) shows the side-view of the test section with a solid wedge inserted.

\section{Results and analysis}

The first set of simulations for each Mach number was initialized such that the reflection configuration was irregular. Therefore, a wedge angle of $25^{\circ}$ was chosen for the initial 


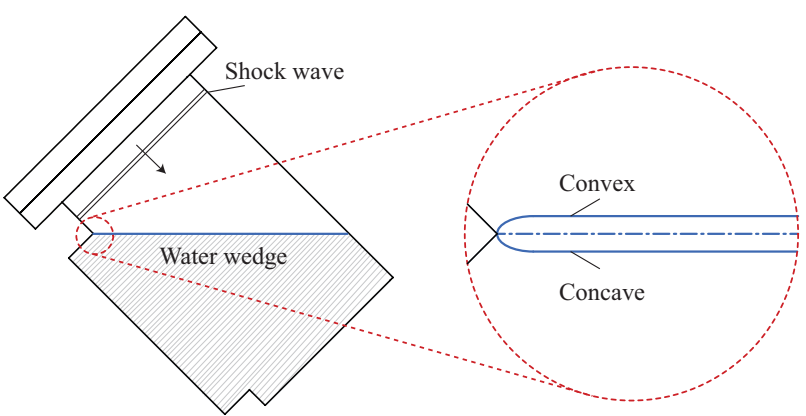

(a)

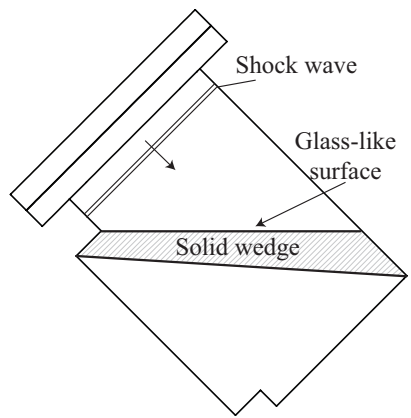

(b)

Figure 7. Side-view of the test section showing a schematic description: (a) a water; (b) a solid wedge.

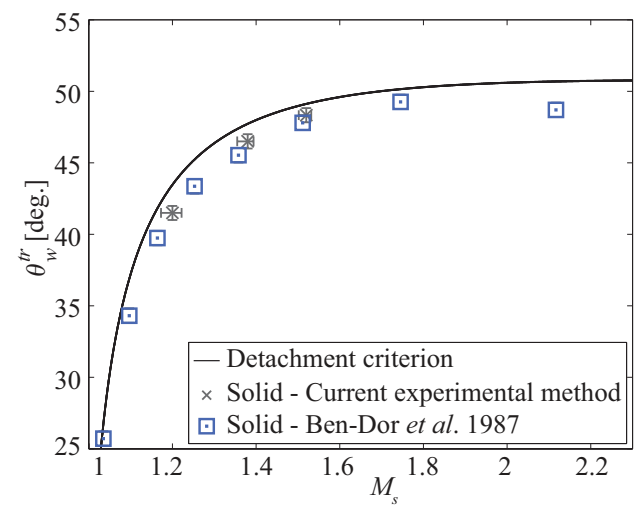

Figure 8. (Color online) The transition angle measured by experimental method versus the incident shock Mach number compared to the previous results (Ben-Dor et al. 1987). The size of the marker size represents the uncertainty of the previous study.

configuration. Then, the inclination angle was increased by steps of $1^{\circ}$ to determine at which angle the Mach stem and slip stream disappear and regular reflection occurs.

\subsection{Validation of the experimental methodology}

The experimental results obtained using the experimental method from the solid surface are validated against the previous experimental data. Figure 8 shows a comparison of the current experimental study and the previous experimental results (Ben-Dor et al. 1987). A comparison to the previous data was made in terms of the transition angle for hydraulically smooth surface case. As it can be seen, a good agreement has been found for the transition angles. Therefore, based on the comparison between the previous and current results, the methodology in this study is able to ensure confidence in the accuracy for finding transition angles.

\subsection{Comparison with experimental results}

A direct comparison between schlieren visualizations obtained in the simulation and experiment with $M_{s}=1.38$ and $\theta_{w}=43^{\circ}$ is shown in figure 9. Single-Mach reflection with a short Mach stem was observed in the simulation, while regular reflection was observed in the experiment. In the experiment, the thickness of the dark band representing the shock wave and the air-water interface that are obtained by using schlieren photography 


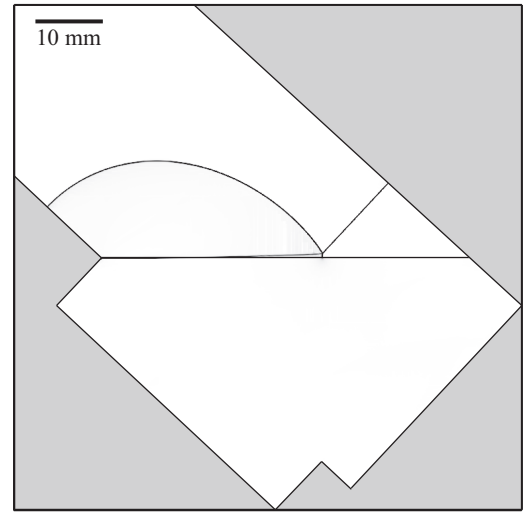

(a) Simulation

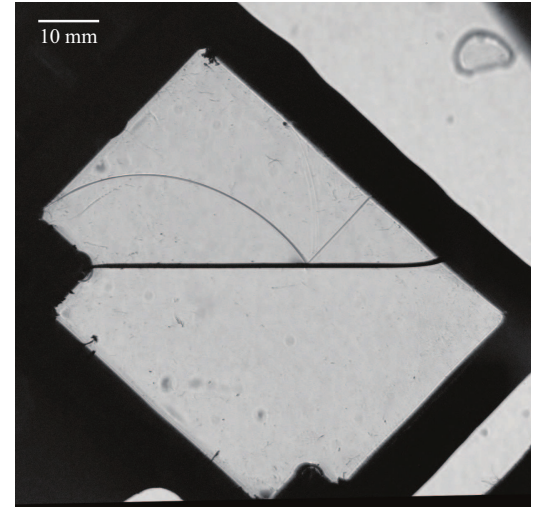

(b) Experiment

FiguRE 9. Comparison of schlieren visualizations for $M_{s}=1.38$ and $\theta_{w}=43^{\circ}$ for (a) simulation, and (b) experiment.

can be affected by several factors such as the strength of the shock wave, a non-parallel incident light angle, and shadowgraph effects. Therefore, the dark band, especially, the air-water interface, looked thicker in the experiment than the simulation. If a Mach stem existed in these experiments, it could be covered by the air-water interface. However, the schlieren visualization for the simulation agrees well with regard to the incident and reflected shock wave locations of that for the experiment.

\section{3. $R R \leftrightarrow I R$ transition angles}

Schlieren images from simulations of different shock reflection configurations at different incident Mach numbers are shown in figure 10. Figure 10(a) shows the single-Mach reflection formed under Mach number $M_{s}=1.52$ and inclination wedge angle $\theta_{w}=25^{\circ}$. Figure 10(b) shows the regular reflection formed using Mach number $M_{s}=1.52$ and wedge angle $\theta_{w}=50^{\circ}$. Figure $10(\mathrm{c})$ shows the regular reflection formed under Mach number $M_{s}=4.0$, and wedge angle $\theta_{w}=50^{\circ}$. The Mach number is much stronger than that used in figure 10(a) and (b). When the shock wave in air with Mach number $M_{s}=3$ or 4 impacts onto the water surface, the impact velocity is on the order of the speed of sound in water, and therefore a shock wave forms in the water and continues to propagate downstream, which is visualized in figures $10(\mathrm{c})$ and $(\mathrm{d})$. Distortion of the water surface can be observed for strong incident shock cases $\left(M_{s}=3\right.$ and 4$)$. As shown in figures $10(\mathrm{c})$ and (d), the water surface near the wedge corner just started to deform, but the deformation is slow compared to the motion of shock wave. When the shock wave in air is weaker, the impact velocity is lower than the speed of sound in water, and thus no shock penetrates into the water region in the cases of figures 10 (a) and (b). In previous experiments with shock wave reflection off water wedges done by Takayama \& Ben-Dor (1989), spherical shock waves that transmitted into a water medium were indeed observed for incident shock Mach numbers 2.33 and 2.30. For lower shock Mach numbers, $M_{s}=1.52$, no spherical shock was observed. Figure $10(\mathrm{~d})$ shows the more complex double-Mach reflection, with an incident Mach number $M_{s}=4.0$ and wedge angle $\theta_{w}=45^{\circ}$.

Single-Mach reflection was observed for lower Mach numbers, $M_{s}=1.20,1.38,1.52$ and 2, while double-Mach reflection was observed for higher Mach numbers, $M_{s}=3$ and 4. In cases of single-Mach reflection, a Mach stem was generated right after the incident 


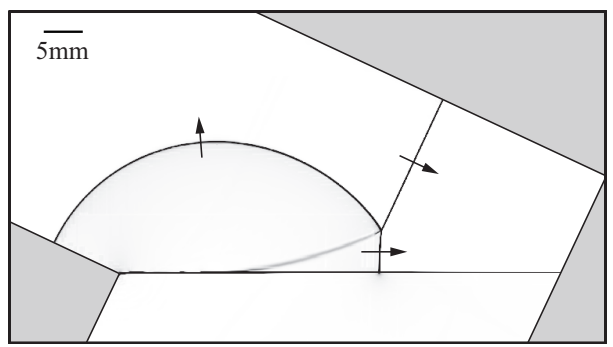

(a)

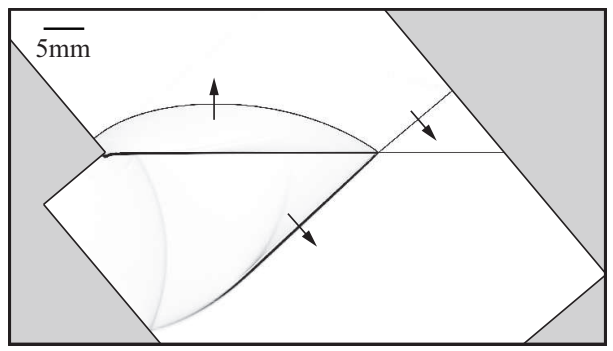

(c)

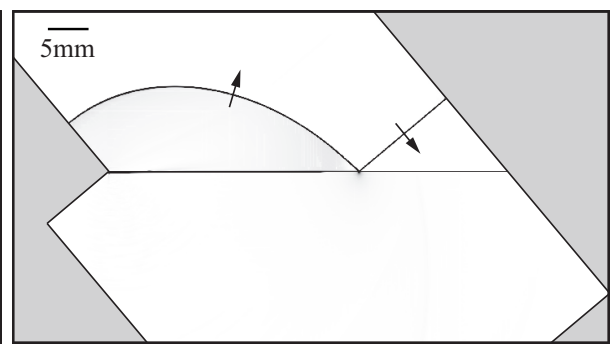

(b)

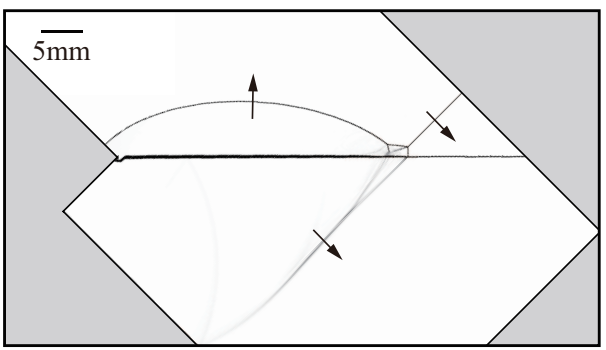

(d)

FIGURE 10. Schlieren visualization showing types of different shock wave reflection configurations off a water wedge. (a) Single-Mach reflection for $M_{s}=1.52$, and $\theta_{w}=25^{\circ}$. (b) Regular reflection for $M_{s}=1.52$, and $\theta_{w}=50^{\circ}$. (c) Regular reflection for $M_{s}=4.0$, and $\theta_{w}=50^{\circ}$. (d) Double-Mach reflection for $M_{s}=4.0$, and $\theta_{w}=45^{\circ}$.

shock reached the water wedge, and the length of the Mach stem kept growing as the incident shock wave travelled further downstream. Simultaneously, a slipstream formed behind the Mach stem.

Figure 11 shows a comparison of the detachment criterion and transition angles for the water and solid wedge cases obtained from the simulations and the experiments. The difference between transition angles obtained from the simulations and those predicted by the detachment criterion is less than $5.3 \%$.

In the simulation, the transition angles for solid wedge cases agreed with the detachment criterion. However, in the experiment, the transition angles for solid wedge were measured $1.5^{\circ}$ lower than the detachment criterion. This is not surprising, since both the simulations of solid wedge cases and the detachment criterion assume inviscid flow and rigid smooth wedge surface and the viscous effects delay the transition from the regular reflection to the Mach reflection (Kleine et al. 2014). The difference between the results of the water wedge cases and the detachment criterion is within $2.7^{\circ}$ in the simulation and $4.5^{\circ}$ in the experiment, which may result from the fact that part of the shock energy was absorbed by water during the reflection. The difference between the simulation and the experiments was mainly caused by the deficiency of viscous effect in the simulation and the thicker air-water interface in the experiment. Another error source for the water wedge cases comes from the use of a multi-component treatment of the volume fractions in the governing equations, see section 2. In addition, some artificial mixing of air and water will invariably occur in cells crossed by the two-fluid interface. While the influence of this effect on the overall solution is mitigated by our use of local mesh refinement, an ad-hoc treatment of the boundary between the two immiscible fluids is unavoidable. Also note that water surface tension is currently not considered in the simulations. 


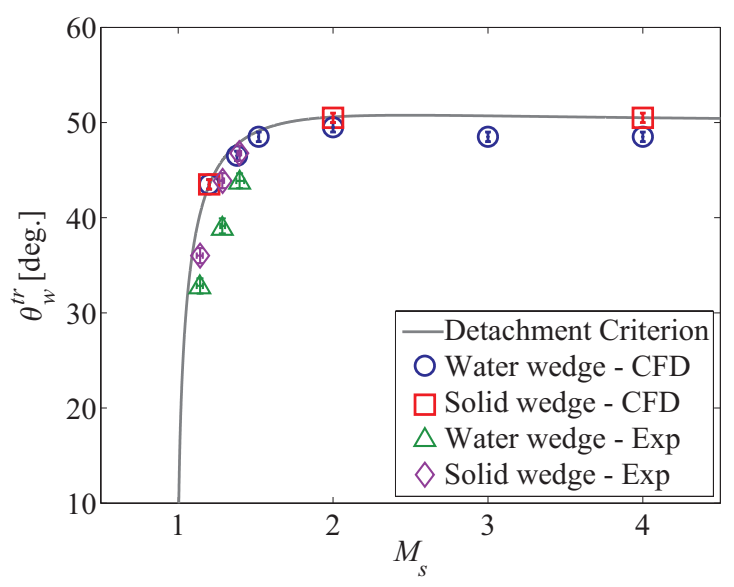

Figure 11. (Color online) Transition angles for water and solid wedge cases observed from simulations and experiments for different incident shock Mach numbers. Detachment criterion (Mouton 2006) plotted for comparison. In the simulation, the transition angles for water wedge cases are measured for six different shock Mach numbers $M_{s}=1.20,1.38,1.52,2,3$ and 4 , while it is measured only for three Mach numbers $M_{s}=1.20,2$ and 4 for the case of a solid wedge. In the experiment, three different shock Mach numbers, $M_{s}=1.20,1.38$, and 1.52, are measured.

\subsection{Comparison between water and solid wedge cases}

A direct comparison of transition angles for the water and solid wedge cases for Mach numbers $M_{s}=1.20,2$ and 4 is shown in figure 11. For Mach number $M_{s}=1.20$, the transition angles of water and solid wedge cases are in the same range $43^{\circ}<\theta_{w}<44^{\circ}$. For Mach numbers $M_{s}=2$ and 4 , the transition angles are in the range $50^{\circ}<\theta_{w}<51^{\circ}$ for solid wedge cases, which fit well for the theoretical solution; while the transition angles are in the range $49^{\circ}<\theta_{w}<50^{\circ}$ and $48^{\circ}<\theta_{w}<49^{\circ}$ respectively for water wedge cases, which are $0.5^{\circ}-1.6^{\circ}$ lower than the theoretical solution.

Schlieren images for the water and solid wedge cases under the same conditions, $M_{s}=3$ and $\theta_{w}=45^{\circ}$, are shown in figure 12 , where (a) shows the reflection off the water wedge at time instant $t=31.5 \mu \mathrm{s}$, and (b) shows the reflection off the solid wedge at time instant $t=23.5 \mu \mathrm{s}$. Specifically, the Mach stems of the above two cases are of the same length. Comparing figures 12(a) with (b) it is clear that under this condition the reflection is double-Mach type for both cases. The first and second triple points were denoted as $T P_{w 1}$ and $T P_{w 2}$ in the water wedge cases, and $T P_{s 1}$ and $T P_{s 2}$ in the solid wedge cases. The two reflection configurations above the reflecting surface have similar shape, but the distance between the two triple points are longer in the solid wedge case. The quantification of the reflection structure can be realized by measurement of the triple point trajectories, and corresponding analysis will be further discussed in Section 4.5.

\subsection{Triple point trajectory}

Setting the compressive corner as the origin, the displacement of the first and second triple points in the horizontal $(w)$ and the vertical $(s)$ directions with incident shock Mach number $M_{s}=4$ is plotted in figure 13. Two different inclination angles $\left(\theta_{w}=40^{\circ}\right.$, and $45^{\circ}$ ) were considered for both solid and water wedge cases. As shown in figure 13, the triple point trajectories are straight lines, which corresponds to a linear growth of the Mach stem, as previously reported by Hornung \& Robinson (1982). Figure 13 also shows that $s$ grows faster for lower inclination angles.

Figure 14 shows both the first and second triple point trajectories for shock reflection 


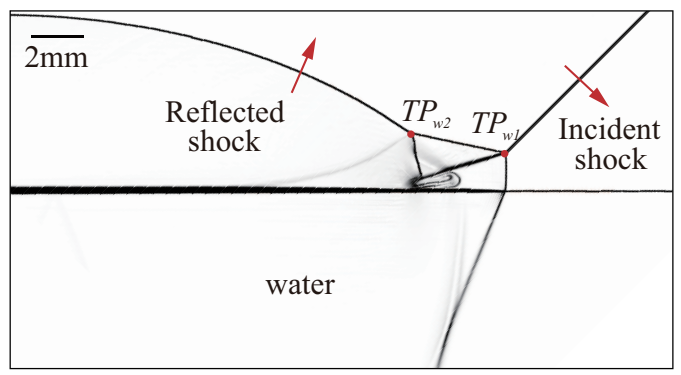

(a) Water wedge

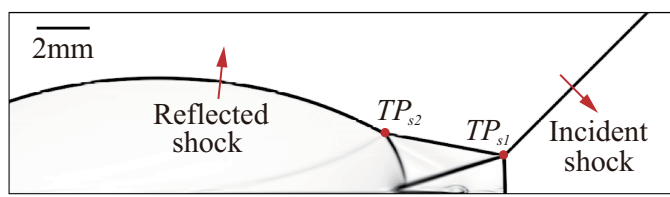

(b) Solid wedge

FIGURE 12. Schlieren visualization of shock wave reflection off (a) a water wedge at time instant $t=31.5 \mu \mathrm{s}$, and (b) a solid wedge at time instant $t=23.5 \mu \mathrm{s}$, with $M_{s}=3$ and $\theta_{w}=45^{\circ}$. The Mach stems in (a) and (b) are of the same length.

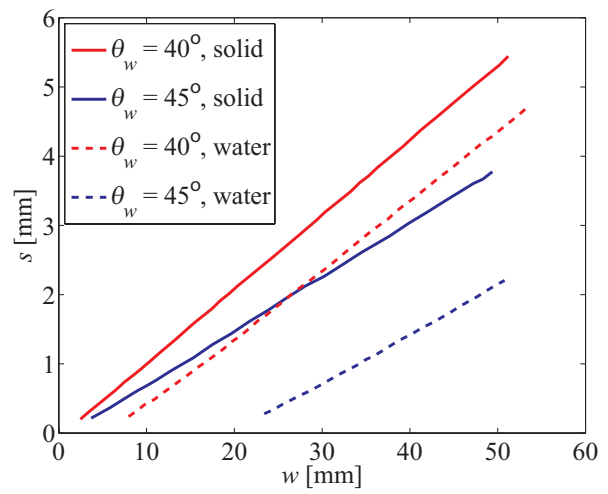

(a) The first triple point

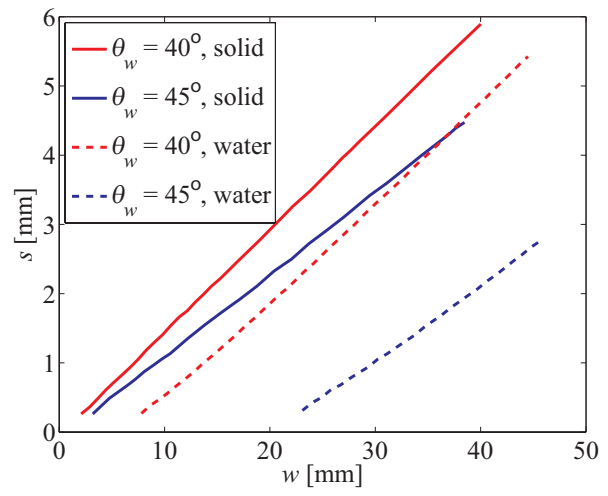

(b) The second triple point

Figure 13. (Color online) The (a) first and (b) second triple point trajectories with Mach number $M_{s}=4$ and two different inclination angles for solid and water wedge cases.

off water wedge with $M_{s}=4$ and $\theta_{w}=40^{\circ}$. At each time instant, the height of the second triple point was always larger than the first triple point.

In the case of double-Mach reflection over the water wedge, both the first and second triple points appeared and started to rise off the surface later than in the cases of the solid wedge with the same $M_{s}$ and $\theta_{w}$. This delay increased for higher values of $\theta_{w}$. One likely reason is that, in the solid wedge cases, no deformation of the surface occurred, where all the energy was reflected at the solid surface during the impact and sustained the growth of the reflection above the solid surface. However, in the water wedge cases, although the air-water interface did not have time to deform in the simulation, part of the energy was absorbed by water during the impact, which reduced the energy reflected from the air-water interface, leading to a reduction of the available energy used to support the growth of Mach stem. Also, vortices were observed near the water surface behind the Mach stem, shown in figure 12(a), which also consumed some of the energy. The vortices 


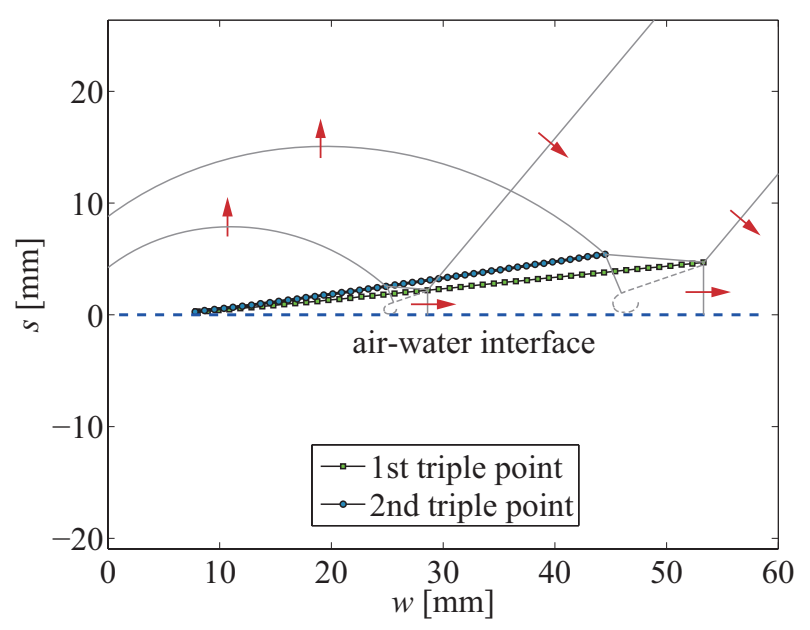

Figure 14. (Color online) The first and second triple point trajectories for shock wave reflection off water wedge with Mach number $M_{s}=4$ and inclination angle $\theta_{w}=40^{\circ}$.

are not artifacts, but are due to the rotational flow. In figure 12, the flow behind the Mach stems are subsonic, and cannot cross the slipstream. In the reference frame of the first triple point, the velocity of the flow near the slipstream is parallel to the slipstream. When the flow reaches the water surface or the solid wedge surface, the flow velocity is forced to be altered to the direction that is parallel to the wedge surface, so rotational flow forms at the foot of the slipstream. As $\theta_{w}$ was increased while keeping $M_{s}$ as constant, the vertical component of the incident shock Mach number increased, which resulted in an increase in the energy transmitted into the water region. Due to the decrease of energy for supporting Mach stem growth, this delay increased as $\theta_{w}$ was increased. Though the establishment of the Mach stem is delayed for the water wedge cases, the growth of the reflection configuration is linear, which can be seen in figures 13 and 14, and therefore the shock reflection process can be described as self-similar.

The triple point trajectory angle was calculated based on linear curve fitting of the trajectory. Figure 15 shows the measurement of the first triple point trajectory angle, $\chi$, from the simulations. The analytic solution from Ben-Dor (1978) was plotted for comparison. For the solid wedge cases, the difference between the measurement and analytic solution is within $1.1^{\circ}$. For the water wedge cases, the difference is less than $0.6^{\circ}$. One likely reason for the difference between solid and water wedge cases is that energy is transmitted into the liquid but not to the solid.

Figure 16 shows the measurement of the second triple point trajectory angle, $\chi^{\prime}$, compared with the analytic solution from Ben-Dor (1980). For the solid wedge cases, the agreement is within $1.3^{\circ}$. For the water wedge cases, the difference is less than $0.9^{\circ}$. One possible reason for the deviation is that the analytic solution assumed uniform flow behind reflected shock wave and Mach stems, while this assumption is not true in the simulated cases.

\subsection{Transmitted waves in water}

Figure 17 shows the velocity profile of the transmitted waves in the water under four different incident shock Mach numbers from the simulation. The threshold of the visualized velocity is chosen so that more details of the flow features inside water can be captured, while the flow behind the incident shock wave and Mach stem in the air 


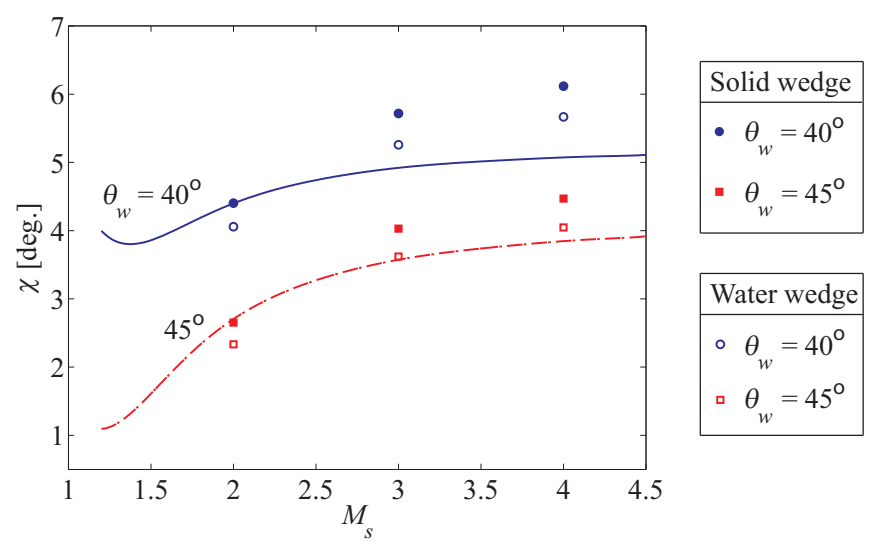

Figure 15. (Color online) The first triple point trajectory angle, $\chi$, for water and solid wedge cases obtained from simulations with different $M_{s}$ and $\theta_{w}$. Analytic solutions from Ben-Dor (1978) are plotted for comparison.

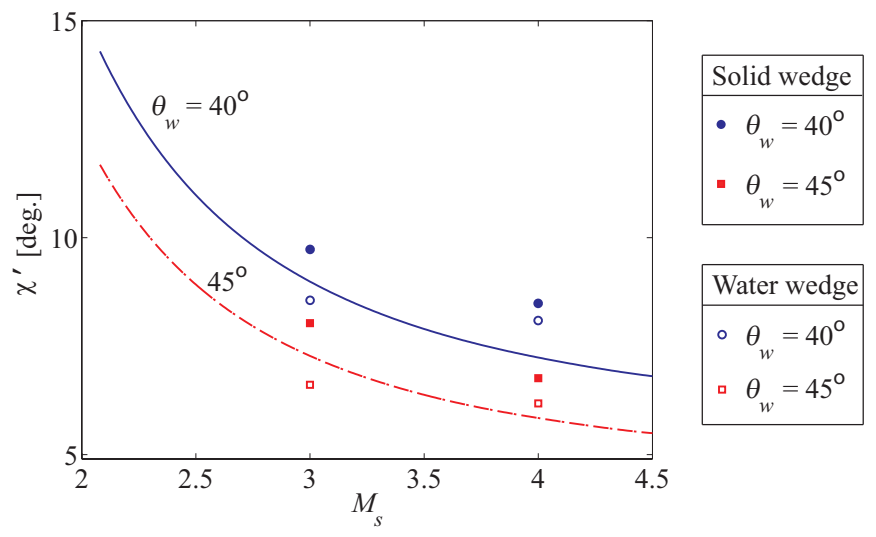

Figure 16. (Color online) The second triple point trajectory angle, $\chi^{\prime}$, for water and solid wedge cases obtained from simulations with different $M_{s}$ and $\theta_{w}$. Analytic solutions from Ben-Dor (1980) are plotted for comparison.

is not visualized. As shown in figures $17(\mathrm{a})$ and (b), the velocity change across the compression wave is smooth, thus the transmitted waves in the water are not shock waves. In particular, in the case of figure 17(a), which shows a regular reflection, the incident shock wave is striking the water surface at an angle of $45^{\circ}$. The foot of the incident shock wave is sliding over the water surface with a uniform speed $v_{i}=587 \mathrm{~m} / \mathrm{s}$. Through calculation, the speed of the compression wave in the water is approximately $v_{t} \approx 1122 \mathrm{~m} / \mathrm{s}$, which is lower than the speed of sound in water $(1452 \mathrm{~m} / \mathrm{s})$. In the case of figure 17(b), illustrsting a single-Mach reflection, the foot of the Mach stem is traversing at the speed $v_{i}=1023 \mathrm{~m} / \mathrm{s}$, while the speed of the compression wave in the water is $v_{t} \approx 1249 \mathrm{~m} / \mathrm{s}$. In the above two cases, the transmitted waves in the water are weak, thus no shock wave is formed in the water. Also, the foot of the incident shock wave or Mach stem cannot catch up with the corner-generated signal traveling in the water, so a precursor wave is induced ahead of the Mach stem that passes the information from water back into the air, as previously reported by Rodriguez et al. (2016). In figures 17(c) and (d), different from (a) and (b), the precursor wave no longer appears. The Mach stems in (c) and (d) are moving with uniform speeds $v_{i}=1575 \mathrm{~m} / \mathrm{s}$ and $2109 \mathrm{~m} / \mathrm{s}$ respectively, 


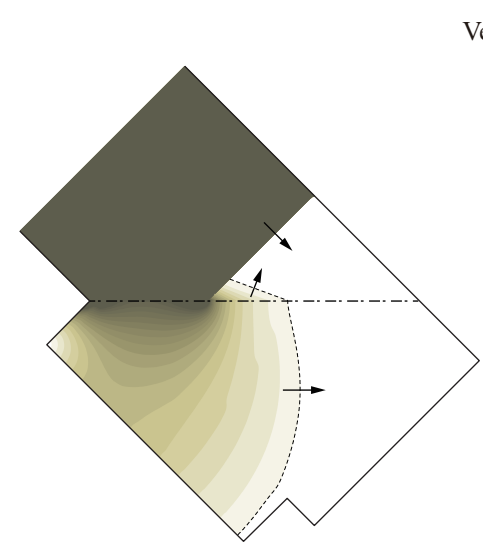

(a) $M_{s}=1.2$



(c) $M_{s}=3$

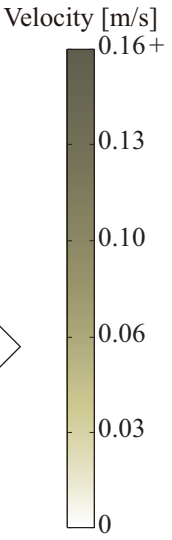

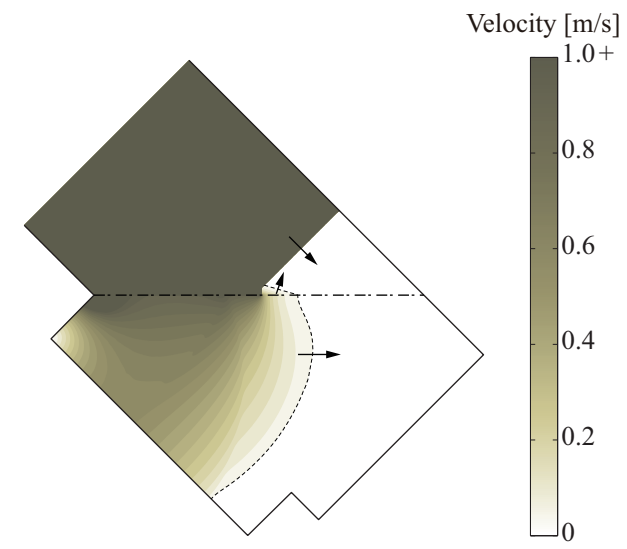

(b) $M_{s}=2$

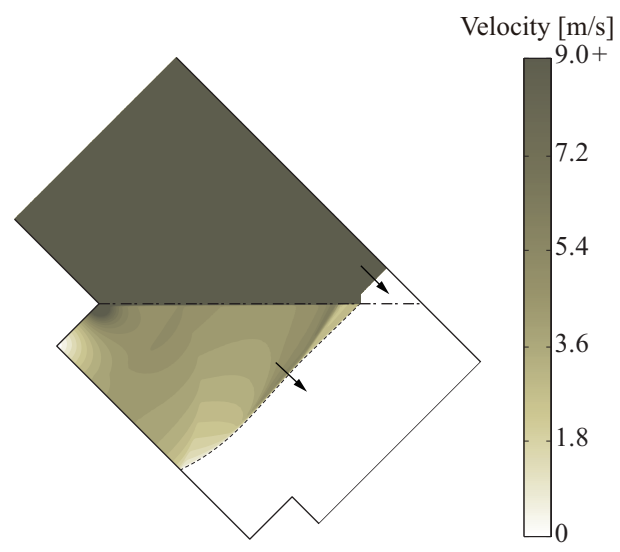

(d) $M_{s}=4$

Figure 17. (Color online) Velocity profile inside water under the incident shock Mach numbers (a) $M_{s}=1.2$, (b) $M_{s}=2$, (c) $M_{s}=3$ and (d) $M_{s}=4$, and wedge angle $\theta_{w}=45^{\circ}$.

which are larger than the speed of sound in water. Thus, a shock wave is formed in the water due to the superposition of the compression waves, and the shock is attached to the Mach stem at every instant.

\subsection{Temperature change in water}

Figure 18 shows the temperature increments in the water for four different incident shock Mach numbers from the simulation. The temperature increment is chosen to be plotted in a certain range so that the changes in the water is clearly visualized, while the temperature field behind the incident shock wave and Mach stem above the airwater interface is not visualized. As shown in figures 18(a) and (b), though the cornergenerated signal propagates fast inside the water, the temperature increment in the water is less than $1 \mathrm{~K}$. In particular, the temperature change for the $M_{s}=1.2$ case is less than $0.1 \mathrm{~K}$. However, in the cases of figures $18(\mathrm{c})$ and (d), a shock wave forms in the water, and a temperature discontinuity is observed across the shock wave. The temperature increment across the shock front in the water is approximately $3.0 \mathrm{~K}$ and 7.3K respectively in figures $18(\mathrm{c})$ and $(\mathrm{d})$. 


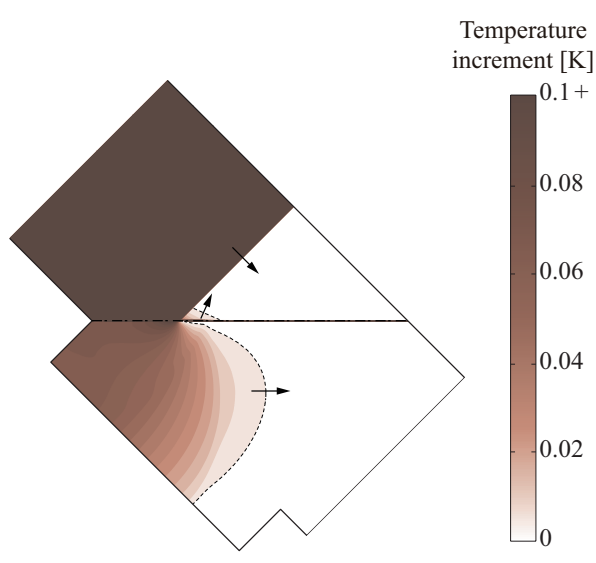

(a) $M_{s}=1.2$

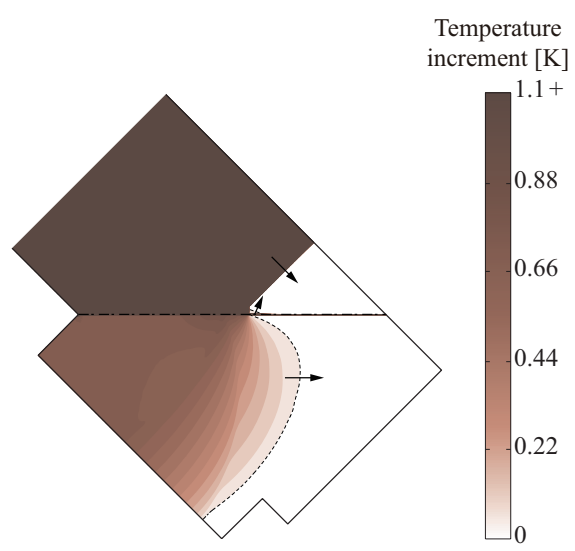

(b) $M_{s}=2$

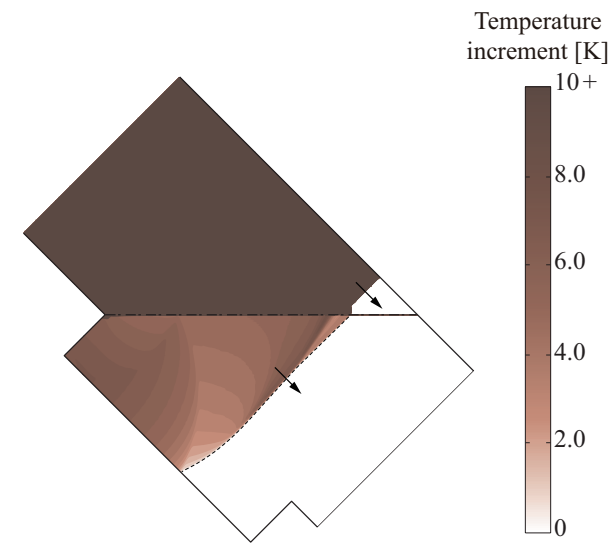

(d) $M_{s}=4$

Figure 18. (Color online) Temperature increment in the water for incident shock Mach numbers (a) $M_{s}=1.2$, (b) $M_{s}=2$, (c) $M_{s}=3$ and (d) $M_{s}=4$, and wedge angle $\theta_{w}=45^{\circ}$.

\section{Conclusions}

In this work, a comprehensive numerical study of shock wave reflection off water wedge was done, supported by experimental results. Different shock wave reflection patterns, transition angles and triple point trajectory angles were studied and compared with reflection off solid wedges.

The major contributions can be summarized as follows. First, high resolution numerical simulations and experiments are performed to study shock wave reflections off water surface. The wave patterns are comprehensively investigated in terms of transition angles and triple point trajectories. Also, simulations are compared against both experimental results and theoretical solutions for the solid wedge cases. The difference between the solid and water wedge cases is analyzed and physically explained. Furthermore, the transmitted waves inside the water are quantitatively studied with regard to the velocity profile and 
the temperature field. Results from the low and high incident shock Mach numbers are compared and two different scenarios are found.

In particular, planar shock wave reflections over water and solid wedges were numerically explored using six different shock Mach numbers, $M_{s}=1.2,1.38,1.52,2,3$, and 4 , and experimentally investigated using three different shock Mach numbers, $M_{s}=1.20$, 1.38 , and 1.52. For both solid and water wedge cases, transition angles show agreement within $5.3 \%$ measured from simulations and $9.2 \%$ measured from experiments comparing with the theoretical detachment criterion for a solid surface.

Moreover, comparing results of the water with solid wedge cases, the transition angle is the same for shock Mach number $M_{s}=1.20$, while the difference gets larger as Mach number increases. Though the establishment of the Mach stem is earlier, the reflection configuration of the solid wedge case has a similar shape as the water wedge case. The difference in wave pattern is primarily due to the energy transmission into the water wedge, which does not exist in the solid wedge case.

The triple point locations for both water and solid wedge cases were traced in the simulation. Results showed that the triple point trajectories are linear. The first and second triple point trajectory angles, $\chi$ and $\chi^{\prime}$, were measured and compared with the analytic solution. Close agreement, within $1.3^{\circ}$ and $0.9^{\circ}$ respectively, was found for the solid and water wedge cases.

The transmitted waves inside the water under four different Mach numbers from the simulations were visualized. Results showed that under relatively low incident shock Mach numbers, $M_{s}=1.2$ and 2 , a precursor wave is induced that passes information from water back into the air. At relatively high Mach numbers, $M_{s}=3$ and 4 , there is no precursor wave, but a shock wave is formed inside the water and it is attached to the Mach stem at every instant.

The temperature field in the water is visualized. For low incident shock Mach numbers, $M_{s}=1.2$ and 2 , the temperature increment in the water is less than $1 \mathrm{~K}$. For the two higher incident shock Mach numbers, $M_{s}=3$ and 4 , the temperature difference across the shock wave in the water is approximately $3.0 \mathrm{~K}$ and $7.3 \mathrm{~K}$, respectively.

The numerical result for the case of incident shock Mach number $M_{s}=1.20$ and inclination wedge angle $\theta_{w}=40^{\circ}$ was compared with the experimental data using schlieren visualization. Results showed good agreement in terms of shock wave configuration.

The authors wish to thank the High Performance Computing Center at University of Southern California for providing free access to computing resources. This study is supported by the National Science Foundation under grant No. CBET-1437412.

\section{REFERENCES}

Abgrall, R. \& Karni, S. 2001 Computations of compressible multifluids. J. Comput. Phys. 169, 594-523.

Baskar, S., Coulouvrat, F. \& Marchiano, R. 2007 Nonlinear reflection of grazing acoustic shock waves: unsteady transition from von Neumann to Mach to Snell-Descartes reflections. J. Fluid Mech. 575, 27-55.

Ben-Dor, G. 1978 Regions and transitions of non-stationary oblique shock wave diffractions in perfect and imperfect gases. UTIAS Rept. No.232.

Ben-Dor, G. 1980 Analytical solution of double-Mach reflection. AIAA Journal. 18(9), 10361043.

Ben-Dor, G. 1981 Relation between first and second triple-point trajectory angles in doubleMach reflection. AIAA Journal. 19(4), 531-533.

Ben-Dor, G. \& Glass, I. I. 1979 Domains and boundaries of non-stationary oblique shockwave reflexions. 1. Diatomic gas. J. Fluid Mech. 92, 459-496. 
Ben-Dor, G. \& Glass, I. I. 1980 Domains and boundaries of non-stationary oblique shockwave reflexions. 2. Monatomic gas. J. Fluid Mech. 96, 735-756.

Ben-Dor, G., Mazor, G., Takayama, K. \& Igra, O. 1987 Influence of surface roughness on the transition from regular to Mach reflection in pseudo-steady flows. J. Fluid Mech. 176, 333-356.

Ben-Dor, G. \& Takayama, K. 1992 The phenomena of shock wave reflection - a review of unsolved problems and future research needs. Shock Waves 2, 211-223.

Ben-Dor, G. 2007 Shock wave reflection phenomena. Springer-Verlag, New York.

Birkhoff, G. 1950 Hydrodynamics, A Study in Logic, Fact and Similitude. Princeton University Press.

Bleakney, W., Weimer, D. K. \& Fletcher, C. H. 1949 The shock tube: a facility for investigations in fluid dynamics. Rev. Sci. Instrum. 20, 807-815.

Borisov, A.A., Kogarko, S.M. \$ Lyubimov, A.V. 1965 Sliding of detonation and shock waves over liquid surfaces. Combust. Explos. Shock Waves 1, 19-23.

Cirak, F., Deiterding, R. \& Mauch, S. P. 2007 Large-scale fluid-structure interaction simulation of viscoplastic and fracturing thin shells subjected to shocks and detonations. Comput. Struct. 85, 1049-1065.

Cole, R. H. 1948 Underwater explosions. Dover.

Colella, P. \& Henderson, L. F. 1990 The von Neumann paradox for the diffraction of weak shock waves. J. Fluid Mech. 213, 71-94.

Deiterding, R. 2009 A parallel adaptive method for simulating shock-induced combustion with detailed chemical kinetics in complex domains. Comput. Struct. 87, 769-783.

Deiterding, R. 2011 Block-structured adaptive mesh refinement-theory, implementation and application. ESAIM: Proceedings 34, 97-150.

Deiterding, R., Cirak, F. \& Mauch, S. P. 2009 Efficient fluid-structure interaction simulation of viscoplastic and fracturing thin-shells subjected to underwater shock loading. In S. Hartmann, A. Meister, M. Schäfer, and S. Turek, eds., Int. Workshop on FluidStructure Interaction. Theory, Numerics and Applications, Herrsching am Ammersee, pp. 65-80. Kassel University Press GmbH.

Deiterding, R., Radovitzky, R., Mauch, S. P., Noels, L., Cummings, J. C. \& Meiron, D. I. 2006 A virtual test facility for the efficient simulation of solid material response under strong shock and detonation wave loading. Eng. Comput. 22(3-4), 325-347.

Delpino Gonzales, O. \& Eliasson, V. 2015 Effect of water content on dynamic fracture Initiation of vinyl ester Exp. Mech. 56, 637-644.

Desjouy, C., Ollivier, S., Marsden, O., Karzova, M. \& Blanc-Benon, P. 2016 Irregular reflection of weak acoustic shock pulses on rigid boundaries: Schlieren experiments and direct numerical simulation based on a Navier-Stokes solver. Phys. Fluids. 28, 027102.

Flåtten, T., Morin, A. \& Munkefjord, S. T. 2011 On solutions to equilibrium problems for systems of stiffened gases. SIAM J. Appl. Math. 71(1), 41-67.

Fox, R.W., McDonald, A.T. \& Pritchard, P. J. 1985 Introduction to fluid mechanics. John Wiley \& Sons New York.

Geva, M., Ram, O. \& Sadot, O. 2013 The non-stationary hysteresis phenomenon in shock wave reflections. J. Fluid Mech. 732, R1.

Grove, J. W. \& Menikoff, R. 1990 Anomalous reflection of a shock wave at a fluid interface. J. Fluid Mech. 219, 313-336.

Henderson, L. F., Ma, J., Sakurai, A. \& Takayama, K. 1990 Refraction of a shock wave at an air-water interface. Fluid Dyn. Res. 5, 337-350.

Hornung, H. G., Oertel, H. \& Sandeman R. J. 1979 Transition to Mach reflexion of shock waves in steady and pseudosteady flow with and without relaxation. J. Fluid Mech. 90, $541-560$.

Hornung, H. G. \& Robinson, M. L. 1982 Transition from regular to Mach reflection of shock waves. Part 2. The steady-flow criterion. J. Fluid Mech. 123, 155-164.

Hornung, H. G. \& Taylor, J. R. 1982 Transition from regular to Mach reflection of shock waves. Part 1. The effect of viscosity in the pseudo-steady case. J. Fluid Mech. 123, $143-153$.

IGRA, D. \& TAKAYAMA, K. 2001 Numerical simulation of shock wave interaction with a water column. Shock Waves 11, 219-228. 
IGRA, D. \& TAKAYAmA, K. 2001 Investigation of aerodynamic breakup of a cylindrical water droplet. Atomization Spray 11, 167-185.

Jeon, H., Gross, J. R., Estabrook, S., Koumlis, S., Wan, Q., Khanolkar, G. R., Tao, X., Mensching, D. M., Lesnick, E. J. \& Eliasson, V. 2015 Shock wave attenuation using foam obstacles: does geometry matter? Aerospace 2, 353-375.

Jolgam, S., Ballil, A., Nowakowski, A. \& Nicolleau, F. 2012 On equations of state for simulations of multiphase flows. Proceedings of the World Congress on Engineering Vol III.

Karzova, M.M., Khokhlova, V.A., Salze, E., Ollivier, S. \& Blanc-Benon, P. 2015 Mach stem formation in reflection and focusing of weak shock acoustic pulses. J. Acoust. Soc. Am. 137, EL436-EL442.

KEDRINSKII, V.K. 2005 Hydrodynamics of Explosion: experiments and models. Springer Berlin Heidelberg, New York.

Kleine, H., Timofeev, E., Hakkaki-Fard, A. \& Sadot, O. 2014 The influence of Reynolds number on the triple point trajectories at shock reflection off cylindrical surfaces. J. Fluid Mech. 740, 47-60.

LAW, C. K. 1970 Diffraction of strong shock waves by a sharp compressive corner. UTIAS Tech. Note No. 150.

LeVeque, R. J. 2002 Finite volume methods for hyperbolic problems. Cambridge University Press, Cambridge, New York.

Li, H. \& Ben-Dor, G. 1995 Reconsideration of pseudo-steady shock wave reflections and the transition criteria between them. Shock Waves 5, 59-73.

MACH, E. 1878 Über den Verlauf von Funkenwellen in der Ebene und im Räume. Sitzungsbr. Akad. Wiss. Wien, 78, 819-838.

Marchiano, R., Coulouvrat, F., Baskar, S. \& Thomas, J.L. 2007 Experimental evidence of deviation from mirror reflection for acoustical shock waves. Phys. Rev. E 76, 056602.

Meng, J. C., Colonius, T. 2015 Numerical simulation of the early stages of high-speed droplet breakup. Shock Waves 25, 399-414.

Mouton, C. A. 2006 Transition between regular reflection and Mach reflection in the dualsolution domain. Ph.D. thesis, California Institute of Technology.

Naidoo, K. \& Skews, B. W. 2011 Dynamic effects on the transition between two-dimensional regular and Mach reflection of shock waves in an ideal, steady supersonic free stream. $J$. Fluid Mech. 676, 432-460.

von Neumann, J. $1943 a$ Oblique reflection of shocks. Explos. Res. Rep. 12, Navy Dept., Bureau of Ordinance, Washington, DC, USA.

von Neumann, J. $1943 b$ Refraction, intersection and reflection of shock waves. NAVORD Rep 203-45, Navy Dept., Bureau of Ordinance, Washington, DC, USA.

Onodera, H. \& TAKayama, K. 1990 Interaction of a plane shock wave with slitted wedges. Exp. Fluids 10, 109-115.

Perotti, L. E., Deiterding, R., Inaba, K., Shepherd, J. \& Ortiz, M. 2013 Elastic response of water-filled fiber composite tubes under shock wave loading. Int. J. Solids Struct. 50, 473-486.

Ram, O., Geva, M. \& Sadot, O. 2015 High spatial and temporal resolution study of shock wave reflection over a coupled convex-concave cylindrical surface. J. Fluid Mech. 768, 219-239.

Ridah, S. 1988 Shock waves in water. J. Appl. Phys 64, 152-158.

Rodriguez, V., Jourdan, G., Marty, A., Allou, A. \& PArisse, J.D. 2016 Planar shock wave sliding over a water layer. Exp. Fluids 57, 125.

Sakurai, A. 1974 Blast wave from a plane source at an interface. J. Phys. Soc. JAP 36, 610-610.

Sasoh, A., Takayama, K. \& Saito, T. 1992 A weak shock wave reflection over wedges. Shock Waves 2, 277-281.

Saurel, R. \& AbGrall, R. 1999 A multiphase Godunov method for compressible multifluid and multiphase flows. J. Comput. Phys. 150, 425-467.

Settles, G. S. 2012 Schlieren and shadowgraph techniques: visualizing phenomena in transparent media. Springer, Berlin.

SHyUE, K.-M. 1998 An efficient shock-capturing algorithm for compressible multicomponent problems. J. Comput. Phys. 142, 208-242. 
SHYUE, K.-M. 1999 A fluid-mixture type algorithm for compressible multicomponent flow with van der Waals equation of state. J. Comput. Phys. 156, 43-88.

Shyue, K.-M. 2006 A volume-fraction based algorithm for hybrid barotropic and non-barotropic two-fluid flow problems. Shock Waves 15, 407-423.

Skews, B. 2005 Shock wave interaction with porous plates. Exp. Fluids 39, 875-884.

Skews, B. W. \& BlitterswiJk, A. 2011 Shock wave reflection off coupled surfaces. Shock Waves 21, 491-498.

Skews, B. W. \& Kleine, H. 2010 Shock wave interaction with convex circular cylindrical surfaces. J. Fluid Mech. 654, 195-205.

Soni, V., Hadjadj, A., Chaudhuri, A. \& Ben-Dor G. 2017 Shock-wave reflections over double-concave cylindrical reflectors. J. Fluid Mech. 813, 70-84.

TAKAyAma, K. \& Ben-Dor, G. 1989 Pseudo-steady oblique shock wave reflections over water wedges. Exp. Fluids 8, 129-136.

Teodorczyk, A. \& Shepherd, J.E. 2012 Interaction of a shock wave with a water layer. Technical Report No. FM2012-002. Graduate Aeronautical Laboratories, California Institute of Technology.

Toro, E. F., Spruce, M. \& Speares, W. 1994 Restoration of the contact surface in the HLL-Riemann solver. Shock Waves 4, 25-34.

Versluis, M. 2013 High-speed imaging in fluids. Exp. Fluids 54, 1458.

Wang, C. \& Eliasson, V. 2012 Shock wave focusing in water inside convergent structures. Int. J. Multiphysics 6, 267-282. 